\title{
A Scaling Law to Predict the Finite-Length Performance of Spatially-Coupled LDPC Codes
}

\author{
Pablo M. Olmos and Rüdiger Urbanke
}

\begin{abstract}
Spatially-coupled LDPC codes are known to have excellent asymptotic properties. Much less is known regarding their finite-length performance. We propose a scaling law to predict the error probability of finite-length spatially-coupled code ensembles when transmission takes place over the binary erasure channel. We discuss how the parameters of the scaling law are connected to fundamental quantities appearing in the asymptotic analysis of these ensembles and we verify that the predictions of the scaling law fit well to the data derived from simulations over a wide range of parameters. The ultimate goal of this line of research is to develop analytic tools for the design of spatially-coupled LDPC codes under practical constraints.
\end{abstract}

Index Terms - codes on graphs, spatially-coupled LDPC codes, iterative decoding thresholds, finite-length code performance.

\section{INTRODUCTION}

$\mathbf{R}$ ECENTLY, it has been proven that spatially-coupled low-density parity-check (SC-LDPC) codes achieve the capacity of binary-input memoryless output-symmetric (BMS) channels under iterative decoding [1]. An SC-LDPC code is constructed by coupling a chain of $L$ disjoint, or uncoupled, LDPC block codes, each one of length $M$ bits, together with appropriate boundary conditions. $L$ is referred to as the SCLDPC chain length. Since spatial coupling is equivalent to introducing memory into the encoding process, SC-LDPC codes can be viewed as a type of (terminated) LDPC convolutional code (LDPC-CC) [2], [3].

Due to the termination, the Tanner graph of an SC-LDPC code has a so-called "structured irregularity", where paritycheck nodes located at the ends of the chain are connected to a smaller number of variable nodes than those in the middle [4]. As a result, the nodes at the ends of the graph form strong subcodes and the resulting reliable information generated there during BP decoding propagates in a wave-like fashion from the ends towards the center. When $M$ tends to infinity and $L$ is sufficiently large, the SC-LDPC code ensemble exhibits a BP threshold very close to the maximum-a-posteriori (MAP) threshold of the uncoupled LDPC code ensemble [4], [5]. An

Pablo M. Olmos is with the Dept. Teoría de la Señal y Comunicaciones, Universidad Carlos III de Madrid, Avda. de la Universidad 30, 28911, Leganés (Madrid), Spain. E-mail: olmos@tsc.uc3m.es

Rüdiger Urbanke is with the School of Computer and Communication Sciences, École Polytechnique Fédérale de Lausanne, Switzerland. E-mail: ruediger.urbanke@epfl. ch

This work was supported by the European project STAMINA, 265496, by Spanish government MEC TEC2012-38800-C03-01, and by Comunidad de Madrid (project 'CASI-CAM-CM', id. S2013/ICE-2845.

This paper was presented in part at 2013 IEEE Information Theory Workshop, Sevilla, Spain.

Copyright (c) 2014 IEEE. Personal use of this material is permitted. However, permission to use this material for any other purposes must be obtained from the IEEE by sending a request to pubs-permissions@ieee.org. added feature of SC-LDPC codes is that the Tanner graph retains the structure and properties of the uncoupled graph. For instance, if the uncoupled LDPC code ensemble has a linear growth of the minimum distance as a function of the block length as is the case for regular LDPC code ensembles, this property is maintained for the SC-LDPC code ensemble [3], [6], [7].

Multiple families of SC-LDPC codes have been proposed and analyzed to date. In [1] and [5], the authors consider a family of codes generated by coupling together a series of $(l, r)$-regular LDPC codes using a random pattern, determined by the so-called smoothing parameter $w$. Coupling the codes following a randomized procedure is convenient for the asymptotic analysis in terms of density evolution (DE). In the limit $w \rightarrow \infty$, it was proven that the BP threshold of the SCLDPC code ensemble converges to the MAP threshold of the uncoupled $(l, r)$-regular LDPC code ensemble. Alternatively, we can construct SC-LDPC codes with a certain predefined coupling structure by means of protographs [8], defining a particular class of multi-edge type (MET) ensemble. It has been shown that the inherent structure in protograph-based SC-LDPC code ensembles can improve minimum distance properties [4], [6], [9]. Also, the predefined structure allows the use of efficient low-delay windowed decoding schemes [10].

In light of all the above, it is probably fair to state that the asymptotic analysis of SC-LDPC code ensembles is well understood. Much less is known about their finite-length behavior in the waterfall region [5]. Even though there are multiple recent papers reporting simulation results for different classes of finite-length SC-LDPC codes [11], [12], [13], [14], [15], to date we lack analytical models to relate the $\mathrm{BP}$ block error probability of finite-length SC-LDPC codes to their structural parameters. The present paper addresses this problem by extending existing analytical results to analyze finite-length LDPC codes [16], [17] to a particular family of SC-LDPC codes.

We consider a SC-LDPC code ensemble that is more structured than the purely random ensemble in [5] but less structured than the protograph ensemble. We denote the ensemble considered here as the $(l, r, L)$ ensemble. Why do we consider this particular ensemble? It has been observed empirically that a proper structure improves performance. But structure also makes the analysis harder since it typically involves more parameters. The chosen ensemble has a good performance while at the same time is still manageable in terms of complexity. It is therefore a good compromise. But we note that the same type of finite-length analysis can in principle 
be also performed for other spatially-coupled code ensembles, and in particular for protograph-based ensembles (using more involved calculations). Further, simulation results suggest that the form of the proposed expression to predict the performance of the $(l, r, L)$ ensemble also applies to protograph-based SC-LDPC code ensembles if some parameters are adjusted appropriately [18].

In [16], [19], the authors analyze the finite-length performance over the binary erasure channel (BEC) of $(l, r)$-regular LDPC code ensembles in the waterfall region. For the erasure channel, the workings of the BP decoder can be cast in an alternative formulation, namely as peeling decoder (PD) [20]. In this formulation, any time the value of a variable node is determined, this variable node and all attached edges are removed from the graph. In this way we get a sequence of residual graphs. Variable nodes are determined either due the received word or later, during the decoding process, if they are connected to a degree-one check node. The analysis of the decoding process consists in studying the statistical evolution of the residual graph as a function of time. Indeed, it suffices to analyze the evolution of the degree distribution (DD) of the residual graph, since this constitutes a sufficient statistic [21].

As shown in [16], the DD of the residual graph at any time converges (in the code length) to a multivariate Gaussian whose mean and covariance matrix is given by the solution of a coupled system of differential equations. Using these results, estimating the error probability consists in computing the probability that, during the decoding process, the random process representing the fraction of degree-one check nodes in the residual graph reaches zero before all variables have been determined. For the $(l, r)$-regular LDPC code ensemble, the error probability is dominated by the statistics of such a process at a single critical point in time. This critical time is the time at which the expected fraction of degree-one check nodes in the graph takes on a local minimum [21].

In the present work we extend the described methodology to the $(l, r, L)$ ensemble. We derive a system of differential equations that characterize the statistics of the DD of the residual graph at any time during the PD process. In particular, the solution of this system provides the expected number of degree-one check nodes and the variance around the expected value at any time during the decoding process. In contrast to $(l, r)$-regular LDPC code ensembles, we show that for the $(l, r, L)$ ensemble there exists a critical phase during which both the expected fraction of degree-one check nodes in the graph and its variance are constant over time. At any time during the critical phase, the decoder might fail with uniform probability. We then proceed in estimating the cumulative error probability during this critical phase. This requires some effort since the DD evolution is correlated over time. More specifically, we show that during the critical phase the covariance between the fraction of degree-one check nodes at two different decoding time instants $\tau$ and $\zeta$ decays exponentially with $|\tau-\zeta|$. Furthermore, the rate of decay is governed by the $(l, r, L)$ coupling pattern.

Under the assumption that the distribution of the number of degree-one check nodes in the graph is Gaussian [16], the statistics for the fraction of degree-one check nodes during the critical phase of the decoding process are those of an appropriately chosen Ornstein-Uhlenbeck (OU) process [22]. OU processes have been widely studied and used in diverse areas of applied mathematics like biological modeling [23], [24], mathematical finance [25], [26] and statistical physics [27]. Using known results for the statistical distribution of the first time at which an OU process is above certain threshold [28], [27], [29], we propose a closed-form expression to estimate the error probability of the SC-LDPC code as a function of $M, L$ and the gap to the ensemble's BP threshold. We illustrate the accuracy of the obtained scaling law by comparing with simulated error probability curves. Further, the presented closed-form scaling law is consistent with the behavior we observed by simulations in [15], capturing the right scaling behavior between $L, M$ and the block error rate. The results presented in this paper contribute to a better understanding of the performance of finite-length SCLDPC codes and the proposed scaling law constitutes a useful engineering tool for code design. In particular, it can be used to accurately estimate the performance improvement/degradation when a certain parameter of the SC-LDPC code is modified.

The paper is structured as follows. In Section III we review the construction of the SC-LDPC code ensemble that is analyzed in the rest of the paper. In Section III we derive the differential equations that describe the expected graph evolution during PD. Graph covariance evolution is discussed in Section IV In Section V] we present the scaling law to predict the SC-LDPC block error rate and, finally, in Section VI we provide some concluding remarks and potential future lines of research.

\section{Construction of The SC-LDPC CODE Ensemble}

Consider a set of $L$ uncoupled $(l, r)$-regular LDPC block codes of length $M$ bits, where $l$ is the variable-node degree and $r \geq l$ is the check-node degree. The Tanner graph of each individual code has $M$ variable nodes, each of degree $l$, and $\frac{l}{r} M$ (assume it to be integer) check nodes, each of degree $r$, and the (design) code rate of each code is $1-\frac{l}{r}$. From this set of codes we construct an SC-LDPC code by permuting edges in such a way that the individual codes become connected (coupled) but maintain their original degree distribution. Let us describe this procedure in more detail.

The SC-LDPC Tanner graph has one position for each of the $L$ uncoupled codes, index these positions by $u, u=1, \ldots, L$. Hereby, position $u$ contains the set of $M$ variable nodes and $\frac{l}{r} M$ check nodes that originally belonged to the $u$-th uncoupled code.

We consider a very simple and regular coupling pattern that is convenient for the purpose of analysis, and we refer to the corresponding ensemble as the $(l, r, L)$ ensemble. A variable node at position $u$ has exactly one connection to a check node at position $u+i, i=0, \cdots, l-1$. For instance, for the case $l=3$, a variable node at position 5 is connected to one check node at position 5 , one check node at position 6 and one check node at position 7 . To maintain this pattern for all bits in the code, note that $l-1$ extra positions, containing exclusively check nodes, are needed at the end of the chain. Consequently, 
the $(l, r, L)$ graph has $D=L+l-1$ positions with $\frac{l}{r} M$ check nodes each. Each check node in the graph has maximum degree $r$ and hence there are $r \times \frac{l}{r} M=l M$ check node sockets per position.

To generate a code from the $(l, r, L)$ ensemble, we proceed as follows. First, we label the set of check node sockets per position from 1 to $l M$ and the set of variable nodes per position from 1 to $M$. Then, for $u=1, \ldots, D$

1) Perform a random permutation of the set $\{1, \ldots, l M\}$, $\pi_{u}=\operatorname{randperm}\{1, \ldots, l M\}$. The permutation is selected with uniform probability among all possible $l M$ ! permutations.

2) Divide $\pi_{u}$ into $l$ disjoint sets of $M$ elements each. They are denoted by $\pi_{u}^{0}, \pi_{u}^{1}, \ldots, \pi_{u}^{l-1}$.

3) For $i=0, \ldots, l-1$, we place $M$ edges in the graph connecting the $M$ variable nodes at position $u-i$ with the $M$ check node sockets in position $u$ with labels containe 11 in the set $\pi_{u}^{i}$. If $u-i<0$ or $u-i>L$, the check node sockets in position $u$ with labels contained in $\pi_{u}^{i}$ are left empty.

From the perspective of the variable nodes, the ensemble looks like the protograph-based ensemble considered in [4]. However, from the perspective of the check nodes, the ensemble is closer to the random ensemble considered in [5]. As mentioned, we chose this ensemble mainly since it is easy to analyze.

\section{A. Ensemble properties and design rate}

Any code in the $(l, r, L)$ ensemble has the following properties:

I) Every variable node at position $u, 1 \leq u \leq L$, is connected via a single edge to a check node at position $u+i$, for $i=0, \ldots, l-1$.

II) If we select at random an edge that is connected to a check node at position $u, l \leq u \leq L$, then the position of the variable node connected to this edge is a random variable uniformly distributed in the interval $\{u-(l-$ 1), $\ldots, u\}$. For $1 \leq u \leq l-1$ and $L+1 \leq u \leq D$, the same statement holds but in the intervals $\{1, \ldots, u\}$ and $\{u-(l-1), \ldots, L\}$ respectively.

III) All check nodes at position $u, l \leq u \leq L$, have degree $r$.

IV) The degree of a check node picked at random at position $u, 1 \leq u \leq l-1$, is a random variable distributed according to a Binomial distribution of $r$ trials and probability of success $u / l$. Similarly, the degree of a check node picked at random at position $u, L+1 \leq u \leq D$, is a random variable distributed according to a Binomial distribution of $r$ trials and probability of success $(L+l-u) / l$.

Properties I), II) and II) are easy to check. Property IV) is a direct consequence of the step 3 ) in the construction of the code described above. For $1 \leq u \leq l-1$, check node sockets in position $u$ with labels in the sets $\pi_{u}^{0}, \pi_{j}^{1}, \ldots, \pi_{j}^{u-1}$ are occupied while check node sockets with labels in the sets

\footnotetext{
${ }^{1}$ In other words, for $m=1, \ldots, M$, we place an edge in the graph that connects the $m$-th variable node in position $u-i$ to the check node socket in position $u$ whose label corresponds to the $m$-th element in the set $\pi_{u}^{i}$.
}

$\pi_{u}^{u}, \pi_{j}^{u+1}, \ldots, \pi_{j}^{l}$ are left empty. This means that, among the $l M$ check node sockets, $(l-u) M$ of them (chosen at random) are left empty. Thus, every check node socket in this position is left empty with probability $(l-u) / l$. Accordingly, the degree of a check node chosen at random in this position is given by a Binomial distribution with $r$ trials and success probability $u / l$.

To compute the design rate of the $(l, r, L)$ ensemble, we need to find the average number of check nodes in the graph connected to at least one variable node. As discussed above, each one of the $\frac{l}{r} M$ check nodes at positions $l, \ldots, L$ are of degree $r$. By property IV), a check node at position $u$, $1 \leq u \leq(l-1)$, is of degree at least one with probability

$$
1-\left(\frac{l-u}{l}\right)^{r}
$$

and thus the average number of check nodes with degree at least one in the graph is given by $\frac{l}{r} M\left(L-(l-1)+2 \sum_{u=1}^{l-1} 1-\left(\frac{l-u}{l}\right)^{r}\right) . \quad$ Therefore, the design rate of the ensemble is

$$
\mathrm{r}=1-\frac{\frac{l}{r} M\left(L-(l-1)+2 \sum_{u=1}^{l-1} 1-\left(\frac{l-u}{l}\right)^{r}\right)}{M L}
$$

$$
=1-\frac{l}{r}\left(1-\frac{l-1}{L}+2 \frac{\sum_{u=1}^{l-1} 1-\left(\frac{l-u}{l}\right)^{r}}{L}\right)
$$

This tends to $1-l / r$, the rate of the uncoupled $(l, r)$-regular LDPC code ensemble, when $L \rightarrow \infty$.

\section{Peeling Decoding And Expected Graph EVOLUTION}

Consider transmission over the BEC and decoding using the peeling decoder [21]. At each step, the PD removes one degree-one check node and its connected variable node, as well as all edges connected to these two nodes. As a result, the PD process gives rise to a sequence of residual graphs. In [20], it is shown that if we apply the PD to elements of an LDPC code ensemble, the expected sequence of graphs or expected graph evolution can be computed by solving a system of coupled differential equations. Note that the expectation is done here with respect to the channel realization as well as with respect to the code ensemble. Note further that standard arguments show that most codes and most channel realizations lead to a decoding behavior that is close to this expected behavior [21]. Therefore, the solution of the differential equation encodes the asymptotic performance, in particular the threshold of the LDPC code ensemble.

In [16], scaling laws (SLs) were proposed to predict the BP finite-length performance for the $(l, r)$-regular LDPC code 
ensemble in the waterfall region ${ }^{2}$ It was also shown how to compute the parameters of the scaling law from the DD of the ensemble. The four most important scaling parameters are (i) the threshold, (ii) the "critical time(s)" of the process, i.e., the time at which the expected number of degree-one check nodes reaches a local minimum, (iii) the expected number of degree-one check nodes at the critical time(s), and (iv) the variance of this quantity at the critical time(s). The first three parameters can be determined by looking at the expected graph evolution. To determine the fourth parameter, a further system of differential equations, dubbed covariance evolution, has to be solved [16]. For $(l, r)$-regular LDPC code ensembles, there is only a single critical time and the error probability can be estimated from the relationship between the expected number of degree-one check nodes and the variance of the same quantity at this point in time.

In the following, we apply a similar line of reasoning to the $(l, r, L)$ ensemble. In Section III-B we compute the expected graph evolution of the $(l, r L)$ ensemble and we discuss the first three scaling parameters (i)-(iii). In Section IV] we derive the system of differential equations of the graph covariance evolution.

\section{A. Description of the $(l, r, L)$ ensemble in terms of degree distribution}

Our aim is to describe the statistical graph evolution of elements in the $(l, r, L)$ ensemble during the decoding process. In the sequel we denote time by $\ell \in \mathbb{N}$ if we measure time in discrete steps and by $\tau$ once we rescale time to pass to the continuous limit in order to write down a differential equation, where $\tau \doteq \ell / M$. Since the PD removes one variable-node per iteration, in average - the erasures are random - we need $\epsilon L M$ iterations to success and thus $\tau \in[0, \epsilon L]$.

At time $\ell$, let $R_{j, u}(\ell)$ be the number of edges that are connected to check nodes of degree $j, j=1, \ldots, r$, placed at position $u, u=1, \ldots, D$, and let $E_{u}(\ell)$ be the total number of edges connected to check nodes at this position. Thus,

$$
E_{u}(\ell)=\sum_{j=1}^{r} R_{j, u}(\ell)
$$

For the $(l, r, L)$ ensemble all variables nodes are of degree $l$ and it suffices therefore to know the number of remaining variable nodes per position at iteration $\ell$. Let this number be denoted as $V_{u}(\ell)$. Note also that the following relation holds

$$
l \sum_{u=1}^{L} V_{u}(\ell)=\sum_{u=1}^{D} E_{u}(\ell)=\sum_{u=1}^{D} \sum_{j=1}^{r} R_{j, u}(\ell) \doteq E(\ell) .
$$

\footnotetext{
${ }^{2}$ The above analysis only captures "large" decoding failures, i.e., decoding failures where a linear fraction of the bits remains undecoded. Such decoding failures are dominant for channel parameters close to the threshold of the code and lead to the characteristic "waterfall"-shape of the error probability curve in this regime. In addition, the decoder can "essentially" succeed but might fail to decode a few remaining bits. This is the dominant failure mode for channel parameters sufficiently away from the threshold and the mechanism for this failure is of an entirely different nature. The resulting shape of the error probability curve in this regime is called the "error floor."
}

\section{B. $(l, r, L)$ expected graph evolution}

Assume that we use samples from the $(l, r, L)$ ensemble to transmit over a BEC with erasure probability $\epsilon$. We initialize the PD by removing from the graph all variable nodes whose value was received through the channel as well as all their edges. Let $\ell=0$ be the state of the system after this initialization. At $\ell=0$, the expected number of variables per position of the graph is

$$
\mathbb{E}\left[V_{u}(0)\right]=\left\{\begin{array}{cc}
\epsilon M, & 1 \leq u \leq L, \\
0, & \text { otherwise }
\end{array}\right.
$$

and the expected value of $R_{j, u}(0)$ is given by

$$
\mathbb{E}\left[R_{j, u}(0)\right]=j \frac{l}{r} M \sum_{m \geq j}^{r} \rho_{m, u}\left(\begin{array}{c}
m \\
j
\end{array}\right) \epsilon^{j}(1-\epsilon)^{(m-j)},
$$

where $\rho_{m, u}$ is the probability that a check node chosen at random from position $u$ in the original $(l, r, L)$ code graph is of degree $m$. The term that multiplies $\rho_{m, u}$ inside the sum in (6) corresponds to the probability that $m-j$ edges are removed from a degree- $m$ check node after PD initialization, hence it becomes a degree- $j$ check node.

According to the description of the $(l, r, L)$ ensemble and the list of properties described in Section II all check nodes at intermediate positions are of degree $r$ and thus, for $l \leq u \leq L$, $\rho_{r, u}=1$ and $\rho_{m, u}=0, m<r$. For $u=1, \ldots, l-1, \rho_{m, u}$ is a Binomial p.m.f. of the form

$$
\rho_{m, u}=\left(\begin{array}{c}
r \\
m
\end{array}\right)\left(\frac{u}{l}\right)^{m}\left(1-\frac{u}{l}\right)^{(r-m)},
$$

and at the opposite boundary the same quantities can be found by symmetry. Namely, for $u=1, \ldots, l-1$

$$
\rho_{m, L+l-u}=\rho_{m, u} .
$$

Define the normalized DD at time $\tau$ as follows

$$
r_{j, u}(\tau) \doteq \frac{R_{j, u}(\ell)}{M} \quad \text { and } \quad v_{u}(\tau) \doteq \frac{V_{u}(\ell)}{M}
$$

and let $\mathcal{G}(\tau)=\left\{\mathcal{G}_{j, u}(\tau)\right\}$ for $u=1, \ldots, D$ and $j=1, \ldots, r+$ 1 be a compact notation for the set of all DD coefficients, where

$$
\mathcal{G}_{j, u}(\tau)=\left\{\begin{array}{cc}
r_{j, u}(\tau) & j \in\{1, \ldots, r\} \\
v_{u}(\tau) & j=r+1
\end{array} .\right.
$$

Also, to keep the notation uncluttered, in the following we write $[r+1]$ to denote $\{1, \ldots, r+1\}$ and $[D]$ to denote $\{1, \ldots, D\}$.

The expected graph evolution of the $(l, r, L)$ ensemble is determined by $\mathbb{E}[\mathcal{G}(\tau)]$ for $\tau \in[0, \epsilon L]$. As shown in [20], [21], the system of differential equations

$$
\frac{\partial \hat{\mathcal{G}}_{j, u}(\tau)}{\partial \tau}=f_{j, u}(\hat{\mathcal{G}}(\tau))
$$

for $u \in[D]$ and $j \in[r+1]$, where

$$
f_{j, u}(\mathcal{G}(\tau)) \doteq \frac{\mathbb{E}\left[\mathcal{G}_{j, u}\left(\tau+\frac{1}{M}\right)-\mathcal{G}_{j, u}(\tau) \mid \mathcal{G}(\tau)\right]}{1 / M},
$$

has a unique solution and, further, the solution for the initial conditions $\hat{\mathcal{G}}_{r+1, u}(0)=\mathbb{E}\left[V_{u}(0)\right] / M$ and $\hat{\mathcal{G}}_{j, u}(0)=$ 
$\mathbb{E}\left[R_{j, u}(0)\right] / M$, given in 5 and $[6$, deviates from the true mean evolution of $\mathcal{G}(\tau)$ by less than $M^{-1}$. In addition, with probability $1-\mathcal{O}\left(\mathrm{e}^{-\sqrt{M}}\right)$, any sample of $\mathcal{G}$ deviates from $\hat{\mathcal{G}}$ by les $\AA^{3}$ than $M^{-1 / 6}$ [21]. Consequently, in the limit $M \rightarrow \infty$, samples of the process $\mathcal{G}(\tau)$ closely follow $\hat{\mathcal{G}}(\tau)$. Note that the function $f_{j, u}(\mathcal{G}(\tau))$ represents the "drift" (expected change) in the components of the DD as a result of one decoding step. The computation of the expectations in 12$)$ for the $(l, r, L)$ ensemble can be found in Appendix A.

To evaluate the probability of successful decoding, we have to estimate the probability that the random process representing the total fraction of degree-one check nodes in the graph, namely

$$
r_{1}(\tau) \doteq \sum_{u=1}^{D} \mathcal{G}_{1, u}(\tau)=\sum_{u=1}^{D} r_{1, u}(\tau)
$$

stays strictly positive until the whole graph has been peeled off. Note that the ensemble BP threshold $\epsilon_{(l, r, L)}$ is defined as the maximum $\epsilon$ for which the expected fraction of degree-one check nodes in the graph, i.e.,

$$
\hat{r}_{1}(\tau) \doteq \sum_{u=1}^{D} \hat{\mathcal{G}}_{1, u}(\tau)=\sum_{u=1}^{D} \hat{r}_{1, u}(\tau)
$$

is strictly positive for any $\tau \in[0, \epsilon L]$.

\section{Solution and comparison for different ensembles}

Before discussing the solution to $\hat{r}_{1}(\tau)$ in (14) for the $(l, r, L)$ ensemble, it is worth showing the corresponding solution for the uncoupled $(l, r)$-regular LDPC code ensemble. In Fig. 1. we represent the evolution of the expected fraction of degree-one check nodes $\hat{r}_{1}(\tau)$ for a $(3,6)$-regular LDPC code ensemble at $\epsilon=0.415, \epsilon=0.429$ and $\epsilon=0.45$. For this code ensemble, $\hat{r}_{1}(\tau)$ as a function of $\tau$ is known in closed-form [20]. The BP threshold of the (3,6)-regular LDPC code ensemble is $\epsilon_{(3,6)}=0.4294$. Observe that the expected evolution has a single local minima or critical point. Indeed, the threshold is that $\epsilon$ parameter where at the critical point the curve is tangent to the x-axis. Above $\epsilon_{(3,6)}=0.4294$, $\hat{r}_{1}(\tau)$ becomes zero before the whole graph has been peeled off. Therefore, at erasure rates above the threshold, with high probability the PD over any element of the $(3,6)$-regular LDPC code ensemble will not succeed [16].

Let us now discuss the spatially-coupled ensemble. In Fig. 2 . we plot the solution for $\hat{r}_{1}(\tau)$ for the $(3,6,50)$ ensemble (a) and for the $(4,8,100)$ ensemble (b) for different $\epsilon$ values.

\footnotetext{
${ }^{3}$ This concentration result is based on the analysis of the evolution of (martingale) Markov processes due to Wormald [30]. In the Wormald method we do not look at a single Markov process but a sequence of such processes parameterized by some quantity $m,\left\{Z^{(m)}(t)\right\}_{m \geq 1}$. The Wormald method consists of showing that, for increasing $m$, with high probability the random variables $Z^{(m)}(t=0), Z^{(m)}(t=1), \ldots$ stay close to an expected evolution and that this evolution is equal to a solution of a differential equation. In our context, the Markov process of interest is the $\mathrm{DD}$ at any time $\ell$ and we consider sequences of processes corresponding to the residual DD of the $(l, r, L)$ ensemble with increasing $M$ values, which motivates using $M$ to normalize the DD in 9 following Wormald's method. Any quantity proportional to $M$ guarantees concentration around the expected graph evolution and thus could be chosen to normalize the DD. For instance, the total code length $\mathrm{n}=M L$ or the expected number of bits per position after PD initialization $\epsilon M$.
}

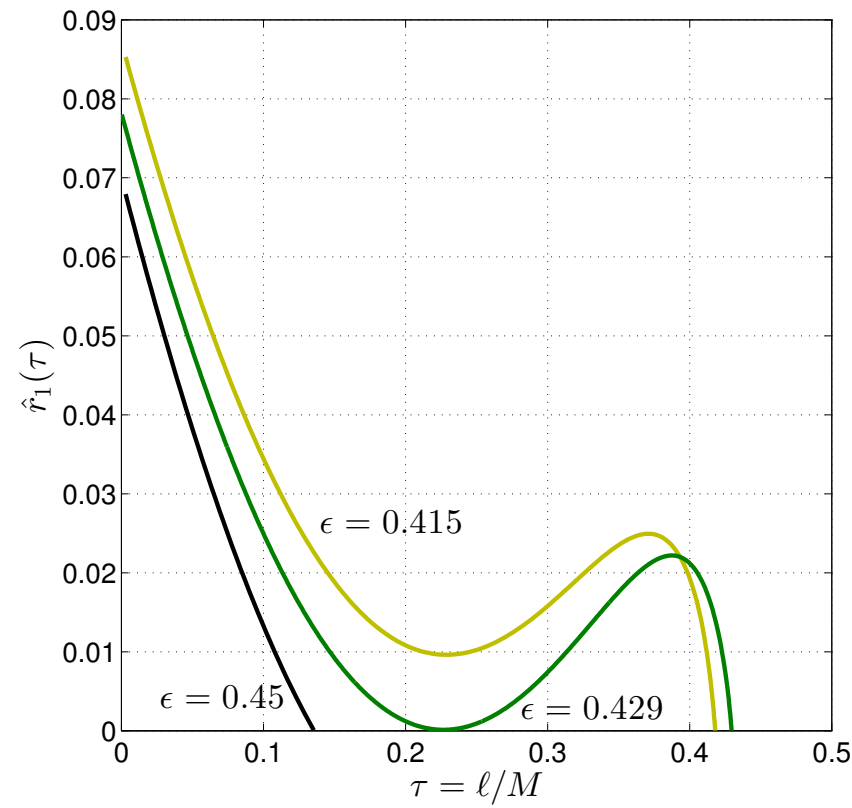

Fig. 1. Evolution of the expected fraction of degree-one check nodes in the residual graph as the PD iterates for the $(3,6)$-regular LDPC code ensemble at $\epsilon=0.415, \epsilon=0.429$ and $\epsilon=0.45$.

The quantity $\hat{r}_{1}(\tau)$ is computed via numerical integration of the differential equations in (11) using Euler's method. For the same ensembles and $\epsilon=0.45$, we also include $\hat{r}_{1}(\tau)$ when the chain length is doubled, i.e., when $L=100$ in (a) and $L=200$ in (b). In Fig. 2(a), for the case $\epsilon=0.45$, we have further included a set of 10 simulated decoding trajectories computed for $M=1000$ bits to show that they indeed concentrate around the predicted evolution.

The evolution of $\hat{r}_{1}(\tau)$ shown in Fig. 2 shows three distinct stages that we now briefly discuss:

1) Initial phase: Similar to what we have seen in Fig. 1 we can observe in Fig. 2(a) and (b) an initial phase of a rapid decay in degree-one check nodes. This phase starts right after the initialization and corresponds to a phase where there are many degree-one check nodes more or less uniformly spread out across the length of the chain. During this phase the bulk of the system behaves essentially like the uncoupled system and only at the boundaries do we see small deviations from this behavior due to the termination. Consequently, degree-one check nodes are removed roughly uniformly along the length of the chain. This can be observed by plotting the average probability $p_{u}(\tau)$ that the PD removes a degree-one check node from position $u$ at time $\tau$ :

$$
p_{u}(\tau) \doteq \frac{\hat{r}_{1, u}(\tau)}{\sum_{m=1}^{D} \hat{r}_{1, m}(\tau)}=\frac{\hat{r}_{1, u}(\tau)}{\hat{r}_{1}(\tau)}
$$

for $u \in[D]$. In Fig. 3, we plot in solid lines the $p_{u}(\tau)$ profile for the $(3,6,50)$ ensemble for $\epsilon=0.45$ at two time instants: $\tau=5(\nabla)$ and $\tau=15(\diamond)$. The dashed lines represent the profile of variable nodes $\hat{v}_{u}(\tau)$ per position, $u=1, \ldots, L$, at the same two instants, $\tau=5(\square)$ and $\tau=15$ 
(o). At $\tau=5$, the decoder is in the initial phase and we can observe that at this point in time $p_{u}(\tau)$ is approximately uniform (with a small extra bump at the boundaries due to the termination). Indeed, based on $p_{u}(\tau)$, we can compute that the cumulative probability of removing a degree-one check node from positions $1-4$ or $48-52$ is less than 0.3 .

2) Second phase: wave-like decoding: If we are transmitting at a channel value which is strictly above the BP threshold of the uncoupled $(l, r)$-regular LDPC code ensemble, then the initial phase ends when all positions except those at the boundaries have run out of degree-one check nodes. At this time, denoted by $\tau^{*}=\tau^{*}(l, r, L, \epsilon)$, the "interior" of the coupled system (i.e., the positions away from the boundaries) has reached a defacto fixed point and this fixed point is equal to the fixed point that the uncoupled system reaches with the same channel parameter [5]. Only towards the two boundaries are there still some degree-one check nodes available and those keep the decoding "alive". In Section III-E we show how to compute a lower-bound on $\tau^{*}$ based on the expected graph evolution for the uncoupled $(l, r)$-LDPC code ensemble.

The second phases starts at $\tau^{*}$ and visually it corresponds to two "decoding waves" that travel at constant constant speed from the boundaries towards the center of the graph [5], [15]. In this phase the degree-one check nodes that are being removed occur mostly around the position where the decoding wave has its rapid rise. This can be seen in Fig. 3 by observing the $p_{u}(\tau)$ and $\hat{v}_{u}(\tau)$ profiles for the $(3,6,50)$ ensemble at $\tau=15$. Note that in this second phase we do not have one critical time point at which the decoder is most likely to stop, but the expected number of degree-one check nodes is essentially a constant throughout this critical phase. Therefore,

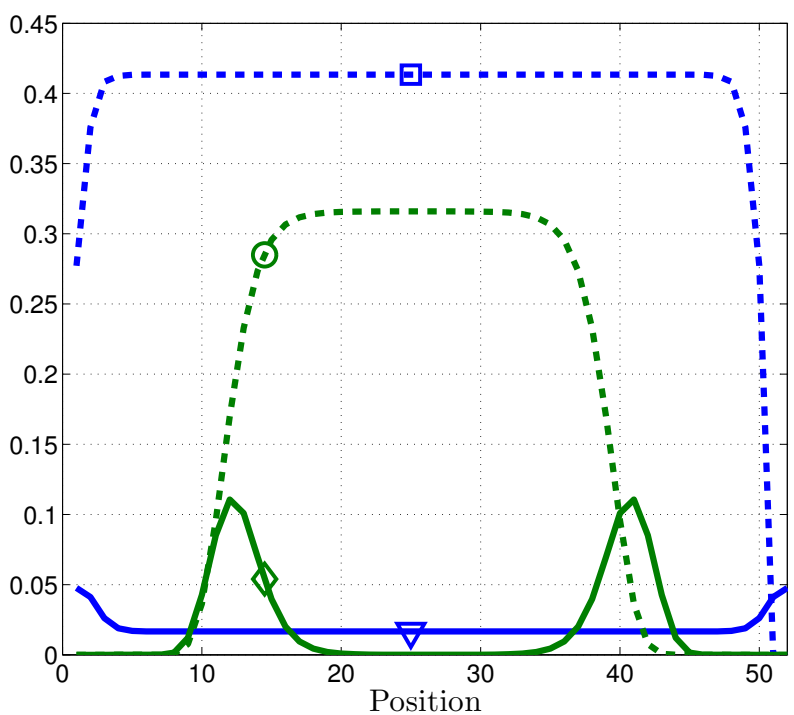

Fig. 3. For the $(3,6,50)$ ensemble, we plot in solid lines the $p_{u}(\tau)$ profile at $\tau=5(\nabla)$ and $\tau=15(\diamond)$ for $\epsilon=0.45$. Dashed lines represent the expected profile of variable nodes per position $\hat{v}_{u}(\tau)$ at the same time instants: $\tau=5$ ( $\square$ ) and $\tau=15$ (०).

we call this the "steady state" phase ${ }^{4}$ Note that the higher we pick $\epsilon$ the closer $\hat{r}_{1}(\tau)$ gets to the zero value. Actually, the $(l, r, L)$ threshold is given by the maximum $\epsilon$ value for which $\hat{r}_{1}(\tau)>0$ during the steady state phase. For the $(3,6,50)$

\footnotetext{
${ }^{4}$ As pointed out in [5], the evolution is not completely flat but exhibits small wiggles. But these wiggles are extremely small and, further, their amplitude vanishes as $l$ tends to infinity. For instance, in Fig. 2 a), the amplitude of the oscillation is $10^{-7}$
}

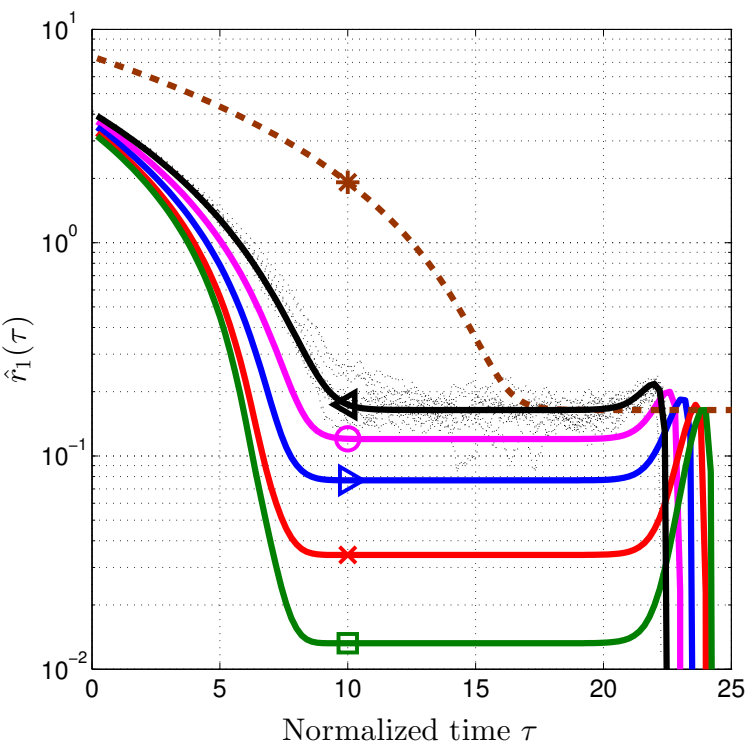

(a)

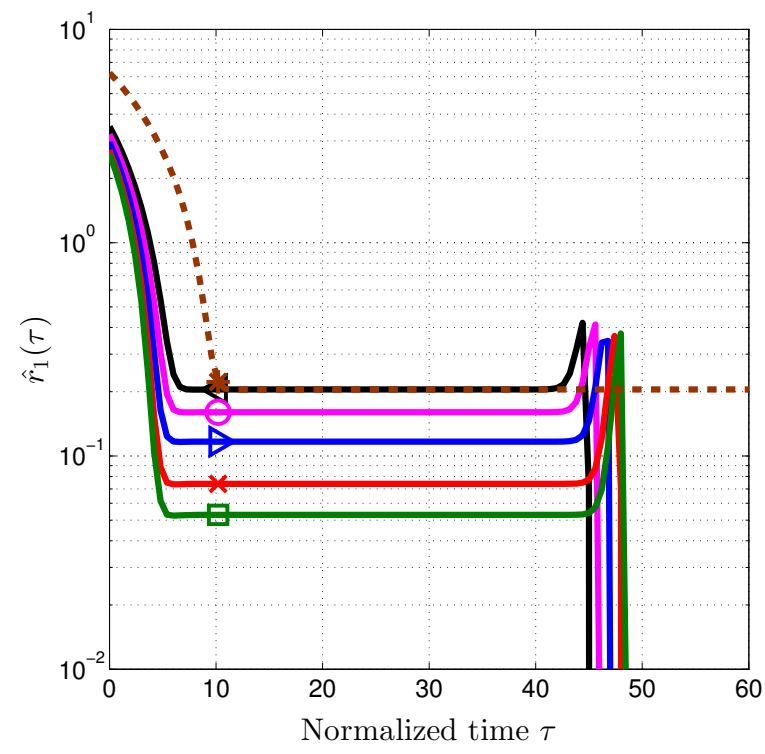

(b)

Fig. 2. $\hat{r}_{1}(\tau)$ in $(14$ for the $(3,6,50)$ ensemble (a) and for the $(4,8,100)$ ensemble (b). In both figures, the $\epsilon$ values considered are: $0.45(\triangleleft), 0.46(\circ)$, $0.47(\triangleright), 0.48(\times)$ and $0.485(\square)$. In (a), for $\epsilon=0.45$ we have included a set of 10 empirical trajectories computed for $M=1000$ bits. They are shown as dashed thin lines. For the same ensembles and $\epsilon=0.45$, we also include $\hat{r}_{1}(\tau)$ for the double chain length, namely $L=100$ and $L=200$ respectively, dashed lines with $(*)$ marker. 
ensemble the BP threshold is $\epsilon_{(3,6,50)}=0.48815$ and for the $(4,8,100)$ ensemble we get $\epsilon_{(4,8,100)}=0.4977$ [4], [5].

It is important at this point to emphasize that we are looking at a spatially-coupled LDPC code ensemble that is terminated at both ends. During the decoding process there is both a decoding wave that moves from the left end towards the middle as well as a decoding wave that moves from the right end towards the middle. Due to these two decoding waves, the expected number of degree-one check nodes is twice what we would get if we considered an ensemble that is terminated only at a single side. The same observation applies to other quantities as well, e.g., the variance of the number of degreeone check nodes that we will compute soon. Rather than aggregating the quantities corresponding to the two waves, we could alternatively think of the decoding process as two processes (the "left" process and the "right" process) and compute the quantities for each of the individual processes separately.

From now on we will stick with the chosen model and leave it to the reader to note the slight modifications that would be necessary if we were to consider ensembles with a one-sided termination only.

3) Third phase: Finally, when the two decoding waves starting at the boundaries meet in the middle of the chain, a third phase takes over. Since the expected fraction of degreeone check nodes in the residual graph at both the first and third phase is significantly larger than in the steady state phase, for most codes in the $(l, r, L)$ ensemble it is very unlikely for the decoder to declare a failure in either the first or the last phase. Getting stuck in the first phase corresponds to an atypical erasure pattern, in which the fraction of erased bits at the boundary is significantly larger than $\epsilon$. This is an unlikely event however, since we know that likely deviations are of the order of the standard deviation and it is proportional to $M^{1 / 2}$. In the last phase, most variable nodes have already been decoded and still the graph contains a large number of degree-one check nodes. For instance, for the $(3,6,50)$ ensemble at $\epsilon=0.45$ and $\tau=22$, we can compute that the expected number of variable nodes in the residual graph is approximately $0.31 M$ while the number of degree-one check nodes is $0.202 M$. Hence, a large fraction of variable nodes can still be decoded. Errors in this regime are typically caused by small cycles in the graph, or stopping sets [21]. As shown in [3], [6], [7], SC-LDPC code ensembles have linear growth of minimum distance with the block length $\mathrm{n}=M L$ and thus codes with no low-weight stopping sets can be easily found for sufficiently large $M$.

In the light of the above, we concentrate on the intermediate steady state phase and inquire how we can express the error probability as a function of the properties of the $r_{1}(\tau)$ random process during this phase.

\section{A closer look at the steady state phase}

Denote by $\hat{r}_{1}(*)$ the expected fraction of degree-one check nodes during the steady state phase. As expected, $\hat{r}_{1}(*) \rightarrow 0$ as the channel parameter approaches the BP threshold. In order to relate the $(l, r, L)$ average block error probability to $\epsilon$, it will be convenient to find the correct scaling of the mean and the variance of the process $r_{1}(\tau)$ in (13) as a function of $\epsilon$. Following [16], we consider a first-order Taylor expansion of both quantities around the ensemble threshold $\epsilon_{(l, r, L)}$. For the case of $\hat{r}_{1}(*)$, we get

$\left.\left.\hat{r}_{1}(*)\right|_{\epsilon} \approx \hat{r}_{1}(*)\right|_{\epsilon_{(l, r, L)}}+\gamma\left(\epsilon_{(l, r, L)}-\epsilon\right)+\mathcal{O}\left(\left(\epsilon_{(l, r, L)}-\epsilon\right)^{2}\right)$.

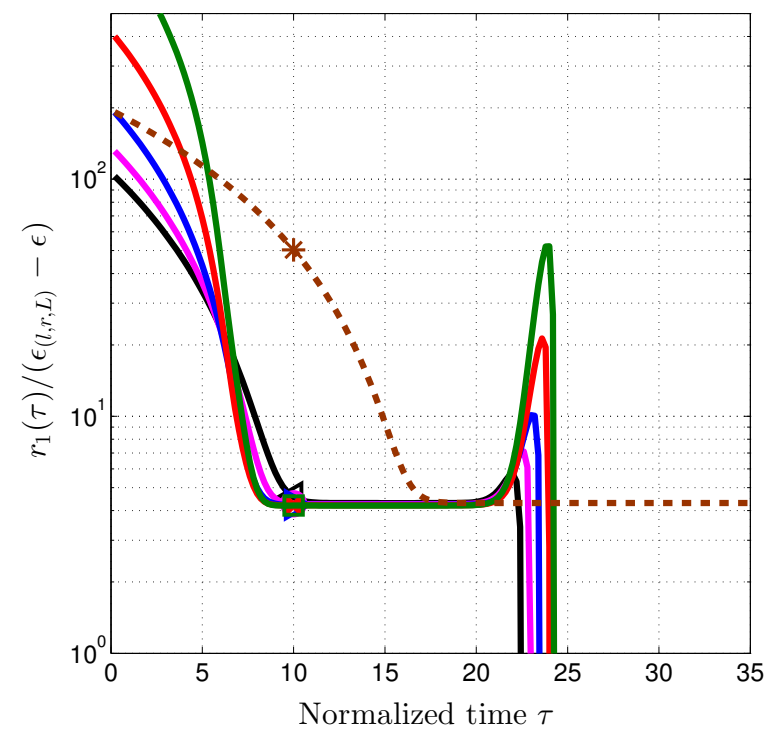

(a)

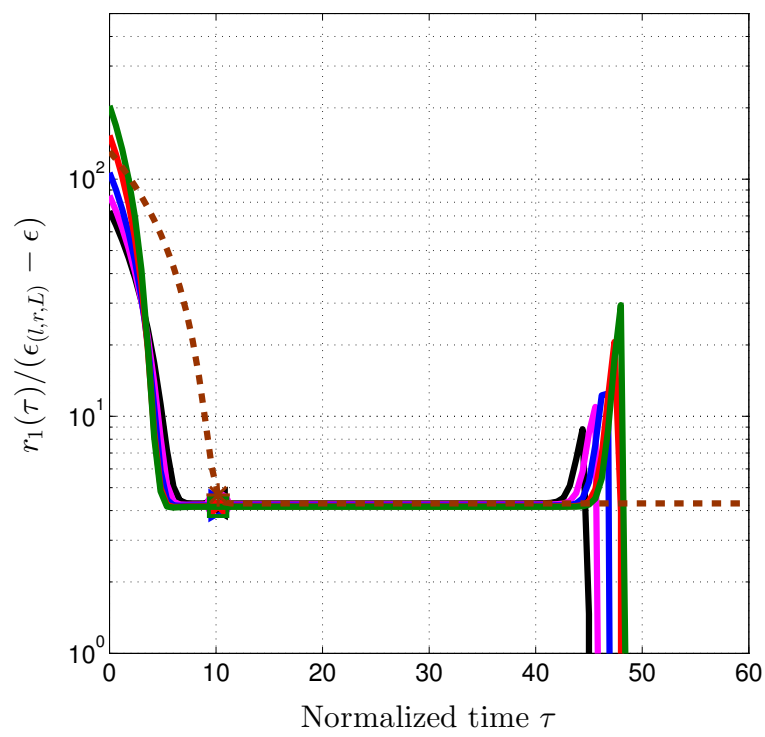

(b)

Fig. 4. We plot $\hat{r}_{1}(\tau) /\left(\epsilon_{(l, r, L)}-\epsilon\right)$ for the $(3,6,50)$ ensemble (a) and for the $(4,8,100)$ ensemble (b). In both figures, the $\epsilon$ considered are: $0.45(\triangleleft)$, $0.46(\circ), 0.47(\triangleright), 0.48(\times)$ and $0.485(\square)$. The thresholds are respectively $\epsilon_{(3,6,50)}=0.48815$ and $\epsilon_{(4,8,100)}=0.49774$. For the same ensembles and $\epsilon=0.45$, we also include $\hat{r}_{1}(\tau) /\left(\epsilon_{(l, r, L)}-\epsilon\right)$ for the double chain length, namely $L=100$ and $L=200$ respectively, dashed lines with $(*)$ marker. 
Since $\left.\hat{r}_{1}(*)\right|_{\epsilon_{(l, r, L)}}=0$ by definition, the $\gamma$ constant can be estimated from the numerical solution to $\hat{r}_{1}(*)$ for a given $\epsilon<\epsilon_{(l, r, L)}$ by

$$
\gamma \approx \frac{\left.\hat{r}_{1}(*)\right|_{\epsilon}-\left.\hat{r}_{1}(*)\right|_{\epsilon(l, r, L)}}{\epsilon_{(l, r, L)}-\epsilon}=\frac{\left.\hat{r}_{1}(*)\right|_{\epsilon}}{\epsilon_{(l, r, L)}-\epsilon} .
$$

In Fig. 4 , we plot $\hat{r}_{1}(\tau) /\left(\epsilon_{(l, r, L)}-\epsilon\right)$ for the two ensembles considered in Fig. 2. Note that this quantity is essentially identical for all $\epsilon$ values in the steady state phase. This indicates that indeed we can ignore the quadratic and higherorder terms in 16 close to the threshold. Hence, we assume the following scaling for the expected fraction of degree-one check nodes during the critical phase

$$
\hat{r}_{1}(*) \approx \gamma\left(\epsilon_{(l, r, L)}-\epsilon\right) .
$$

Further, Fig. 4 confirms, that, as expected, the constant $\gamma$ is the same for the $(l, r, L)$ ensemble and the same ensemble with twice the chain length, i.e., $(l, r, 2 L)$. In Table I] we collect the values of $\gamma$ for various values of $l$ and $r$ computed for $L=$ 100. Also included are the MAP thresholds of the underlying uncoupled $(l, r)$-regular LDPC code ensemble, which are up to numeric precision equal to the threshold $\epsilon_{(l, r, L)}$ for the chosen value of $L$. In all cases, $\gamma$ is computed by evaluating (17) at $\epsilon=\epsilon_{(l, r, L)}-0.04$. 5

TABLE I

$\gamma$ PARAMETER FOR DIFFERENT $(l, r, L)$ ENSEMBLES, ALL WITH $L=100$.

\begin{tabular}{|c|c|c|c|}
\hline 1 & $\mathrm{r}$ & MAP threshold & $\gamma$ \\
\hline 3 & 6 & 0.4881 & 4.31 \\
4 & 8 & 0.4977 & 4.24 \\
5 & 10 & 0.4994 & 4.19 \\
6 & 12 & 0.4999 & 4.15 \\
4 & 12 & 0.3302 & 4.28 \\
5 & 15 & 0.3325 & 4.23 \\
4 & 6 & 0.6656 & 4.2 \\
\hline
\end{tabular}

So far we have seen how to determine $\gamma$ once we solved the differential equation describing the peeling decoder. But $\gamma$ can also be determined via density evolution since it is closely connected to the "speed" of the BP decoder [5], a further quantity of significant practical importance. More precisely, consider the following question. Run a message-passing BP decoder with parallel updates and consider again the "steady phase" of the decoder. How many iterations ${ }^{6}$ does it take until the decoding wave has "moved" by one position? It is natural to define the speed of the decoder to be the inverse of this number. Clearly, the smaller the number of required iterations (the higher the speed) the less complex the decoding is. The number of required iterations is a function of the

\footnotetext{
${ }^{5}$ In principle, we should compute these quantities as close as possible to the threshold. But since we are using numerical integration techniques, it is more stable to consider an $\epsilon$ value that is further away from the threshold of the ensemble. This is particularly important for the integration of the covariance evolution equations described in Section IV This motivated us to choose $\epsilon=\epsilon_{(l, r, L)}-0.04$ as reference $\epsilon$ value to compute the different scaling parameters.

${ }^{6}$ In general it will not take an integral number of iterations to move by exactly one position but rather this number will be fractional.
}

channel parameter and some thought shows that it behaves like $c /\left(\epsilon_{(l, r, L)}-\epsilon\right)$ close to the threshold [31], where $c$ is a real positive constant.

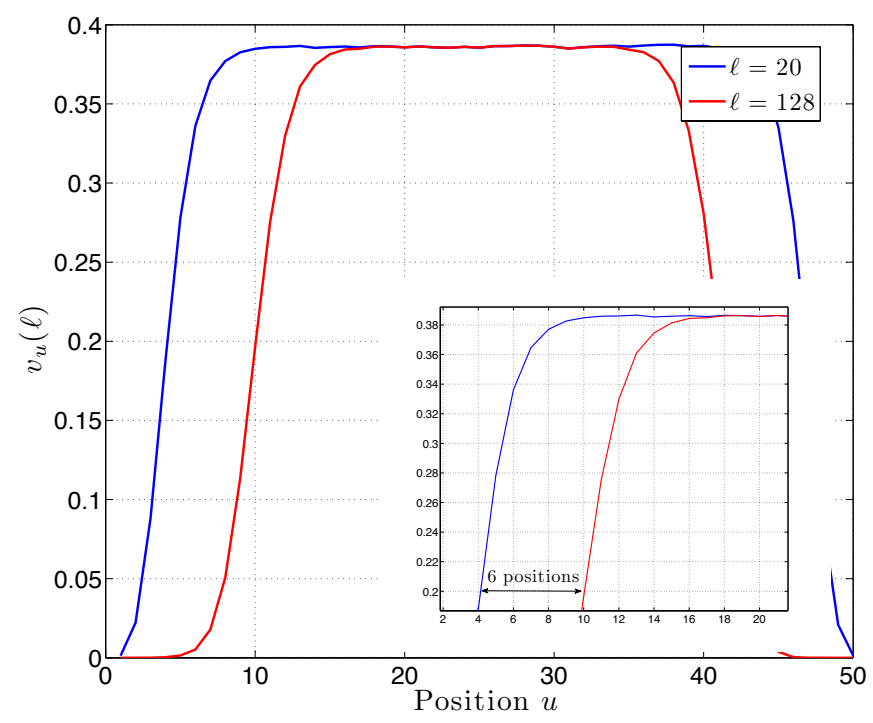

Fig. 5. For a $(3,6,50)$ code with $M=16000$ bits per position, we plot the simulated profile of the fraction of variable nodes left in the graph per position $v_{u}(\ell), u=1, \ldots, L$, after $\ell=20$ and $\ell=128$ iterations of the parallel BP decoder. The channel parameter is $\epsilon=\epsilon_{(l, r, L)}-0.01$. Note that it takes about $128-20=108 \mathrm{BP}$ iterations for the wave to move by 6 positions.

Assume that the decoding wave has moved by exactly one position under the appropriate number of BP steps. How many variable nodes have we determined during these steps? The answer is $2 \beta M$, where $\beta$ is the fraction of yet undetermined variables in the uncoupled system when we transmit at parameter $\epsilon$ and perform an infinite number of iterations (this is the fixed point that we get stuck in when transmitting using an uncoupled code above its BP threshold; the example below will hopefully clarify the exact definition of $\beta$ ). The factor 2 stems from the fact that we are looking at a spatially-coupled ensemble where both ends are terminated and so there is both a "left" wave moving to the right and a "right" wave moving to the left.

Next, note that if $\epsilon_{(l, r, L)}-\epsilon$ is very small then, with high probability, for each variable that we determine there will be exactly one degree-one check node that is connected to it [31]. Finally, by our definition, both the number of check nodes and the number of variable nodes are normalized by $M$. It follows that we must have $\gamma=2 \beta / c$. We note that [32] shows how to compute the speed of the wave for a spatially-coupled Curie-Weiss model and that [31] gives a way to bound the speed of the wave for a particular spatially-coupled LDPC code ensemble. Both of these computations are in terms of quantities that appear in density evolution. It is therefore in principle possible to compute the speed of the wave, and hence $\gamma$, for the $(l, r, L)$ ensemble based on quantities that appear in density evolution.

Example 1: Let us look at one of our running examples, namely the $(3,6,50)$ ensemble. Rather than computing the 
decoding speed analytically, let us determine the speed via simulations. From Fig. 5 we see that it takes about 108 iterations for the decoding wave to move 6 positions, where $\epsilon=\epsilon_{(l, r, L)}-0.01$. Therefore, $c=\frac{108}{6} \cdot 0.01=0.18$. To determine $\beta$, note that for the $(3,6)$ ensemble the BP message-passing decoder gets stuck in the point $x$ when transmitting at parameter $\epsilon$, where $\epsilon(x)=\frac{x}{\left(1-(1-x)^{5}\right)^{2}}$ [21]. With $\epsilon=\epsilon_{(l, r, L)}-0.01=0.47815$ we get $x=0.41475$. We then have $\beta=\epsilon\left(1-(1-x)^{5}\right)^{3}=0.386273$. Therefore, $\gamma=2 \beta / c=4.29$, which is a very good match to the $\gamma$ value in Table I]

We will soon see how $\gamma$ enters in the formula for the error probability but the basic idea is simple. The larger $\gamma$ the more degree-one check nodes we have in expectation at a given value of the channel parameter. Recall that an error occurs if at any point before the whole graph has not been peeled off we run out of degree-one check nodes. In other words, an error occurs if the actual number of degree-one check nodes deviates from the mean and takes on the value zero. So all other parameters (in particular the variance) being equal, the larger the mean, the less likely this event will be.

\section{E. A lower bound on $\tau^{*}$}

The time $\tau^{*}$ at which the steady state phase takes over is a function of the uncoupled $(l, r)$-regular LDPC code ensemble, the coupling pattern, the chain length $L$ and the channel parameter $\epsilon$. A lower bound on $\tau^{*}$, that we denote by $\underline{\tau}$, can be obtained by ignoring the low-rate terminations at both sides of the $(l, r, L)$ code graph and by assuming a $(l, r)$-regular LDPC code ensemble of length $\mathrm{n}=M L$ operating above its BP threshold. The bound $\tau$ is then computed as the expected time at which the graph runs out of degree-one check nodes. This is essentially the same computation that we have performed
TABLE II

$\underline{\tau} / L$ FOR SOME REGULAR ENSEMBLES.

\begin{tabular}{|c|c|c|}
\hline 1 & $\mathrm{r}$ & $\underline{\tau} / L$ \\
\hline 3 & 6 & 0.0814 \\
4 & 8 & 0.0193 \\
5 & 10 & 0.0053 \\
6 & 12 & 0.0015 \\
4 & 12 & 0.020 \\
5 & 15 & 0.0067 \\
4 & 6 & 0.01272 \\
\hline
\end{tabular}

\section{in Example 1.}

Example 2: Let us look again at one of our running examples, namely the $(3,6,50)$ ensemble. As we have seen, when the uncoupled decoder transmits above the BP threshold then the decoder gets stuck before all bits have been decoded and we have denoted the fraction of undecoded bits by $\beta$.

Let us compute $\beta$ when $\epsilon=0.48815$. In this case the fixed point $x$ is equal to $x=0.432261$ and $\beta=\epsilon\left(1-(1-x)^{5}\right)^{3}=$ 0.406764 [21]. Right after the initialization the fraction of uncoded bits is $\epsilon$. Therefore the total number of bits that will have been decoded in a chain of length $L$ (after division by $M)$ is equal to $\tau \doteq L(\epsilon-\beta)$. For the particular case this gives us $L(\epsilon-\beta)=50(0.48815-0.406764)=4.0693$.

In Table [I] we state these bounds for a few examples in the form $7 \underline{7} / L$. For each $(l, r, L)$ configuration, $\beta$ is computed at $\epsilon_{(l, r, L)}$.

\footnotetext{
${ }^{7}$ As we mentioned, at the end of the decoding process, when the two decoding waves "meet", we enter the third phase of the decoding process and during these phase decoding errors are again very unlikely. To get a better approximation to the error probability, one should also try to account for the length of this phase.
}

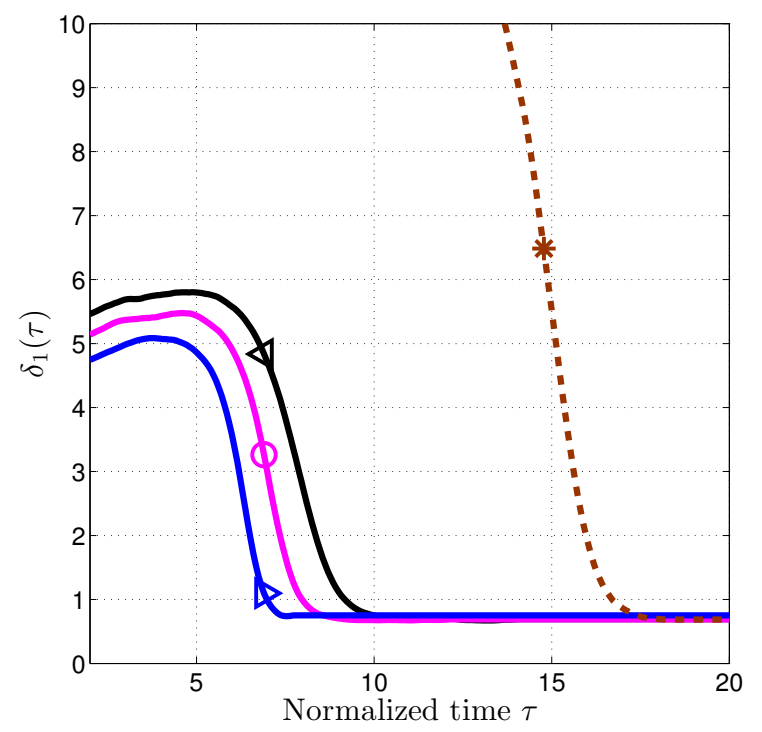

(a)

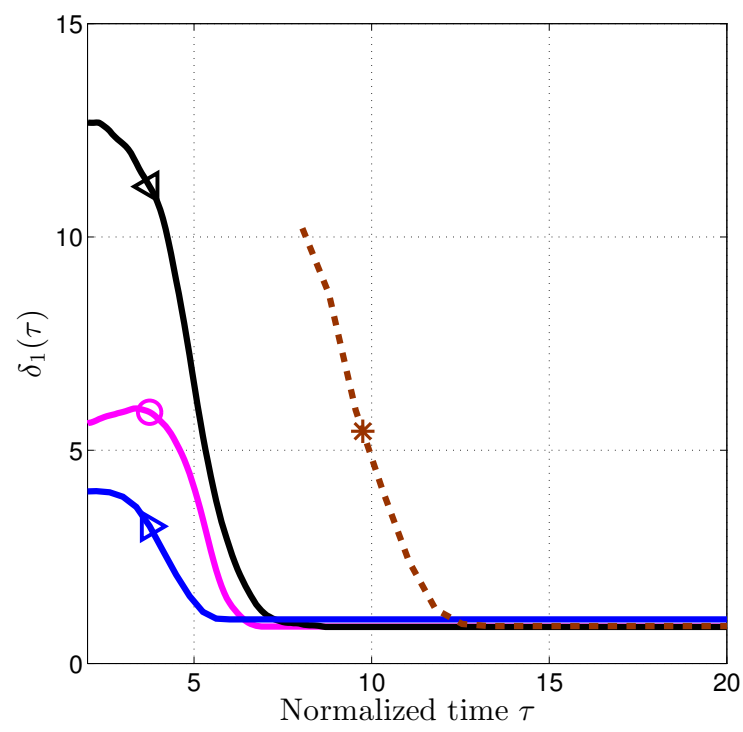

(b)

Fig. 6. In (a), we plot $\delta_{1}(\tau)$ computed for the $(3,6,50)$ ensemble and $\epsilon=0.45(\triangleleft), \epsilon=0.46(\circ)$ and $\epsilon=0.47$ ( $\triangleright$ ). In (b), we reproduce the same results for the $(4,8,100)$ ensemble. In dashed line with $*$ marker, we also plot $\delta_{1}(\tau)$ for $L=100$ (a) and for $L=200$ (b) for $\epsilon=0.45$. 


\section{Covariance Evolution}

Our next goal is to study the second-order statistics of the $r_{1}(\tau)$ process during the steady state phase, which is the main purpose of this section. As shown in [16], the covariance evolution for the $(l, r, L)$ ensemble, i.e., the evolution along peeling decoding of moments of the form $\operatorname{CoVar}\left[\mathcal{G}_{j, u}(\tau), \mathcal{G}_{z, x}(\tau)\right]$ for any pair of positions $(u, x) \in[D]^{2}$ and any pair of degrees $(j, z) \in[r+1]^{2}$, can be estimated by solving an augmented system of differential equations, referred to as covariance evolution. Define

$f_{z, x}^{j, u}(\mathcal{G}(\tau))=$

$\frac{\mathbb{E}\left[\left(\mathcal{G}_{j, u}\left(\tau+\frac{1}{M}\right)-\mathcal{G}_{j, u}(\tau)\right)\left(\mathcal{G}_{z, x}\left(\tau+\frac{1}{M}\right)-\mathcal{G}_{z, x}(\tau)\right) \mid \mathcal{G}(\tau)\right]}{1 / M^{2}}$

If the system of differential equations in (11) is augmented with the set of equations in (19.B), then the solution for $\delta_{z, x}^{j, u}(\tau)$ is also unique and given the initial conditions

$$
\begin{aligned}
\hat{\mathcal{G}}_{j, u}(0) & =\mathbb{E}\left[\mathcal{G}_{j, u}(0)\right] \\
\delta_{z, x}^{j, u}(0) & =\mathbb{E}\left[\mathcal{G}_{j, u}(0) \mathcal{G}_{z, x}(0)\right]-\mathbb{E}\left[\mathcal{G}_{j, u}(0)\right] \mathbb{E}\left[\mathcal{G}_{z, x}(0)\right],
\end{aligned}
$$

the difference with respect the true covariance $\operatorname{CoVar}\left[\mathcal{G}_{j, u}(\tau), \mathcal{G}_{z, x}(\tau)\right]$ at any time $\tau$ is less than $M^{-1 / 2}$ [16]. Further, in the limit $M \rightarrow \infty$ the following holds

- $\mathcal{G}_{j, u}(\tau)$ is Gaussian distributed with mean $\hat{\mathcal{G}}_{j, u}(\tau)$ and variance $\delta_{j, u}^{j, u}(\tau) / M$.

- For any pair of positions $(u, x) \in[D]^{2}$ and any pair of degrees $(j, z) \in[r+1]^{2}, \mathcal{G}_{j, u}(\tau)$ and $\mathcal{G}_{z, x}(\tau)$ are jointly Gaussian distributed with cross covariance $\delta_{z, x}^{j, u}(\tau) / M$.

Note that any covariance moment vanishes in the limit $M \rightarrow \infty$ and hence $\mathcal{G}(\tau)$ concentrates around the mean $\hat{\mathcal{G}}(\tau)$ predicted by $(11)$. The details of the computation of the correlations in 19 can be found in Appendix B In Appendix C, we derive the initial conditions in (21) for the $(l, r, L)$ ensemble. The derivatives of the function $f_{j, u}(\mathcal{G}(\tau))$ with respect to any component $\mathcal{G}_{q, m}(\tau)$, used in (19.B), can be computed given the closed-formed expression to $f_{j, u}(\mathcal{G}(\tau))$ derived Appendix A.

To estimate the error probability of the $(l, r, L)$ ensemble, we need to evaluate the variance of the process $r_{1}(\tau)$ in 13 , whose expected evolution has been described in Section III-C. In the light of the above results, for sufficiently large $M$ the distribution of $r_{1}(\tau)$ is well approximated by a Gaussian process whose variance can be obtained given the solution to the covariance evolution equations in (19.B):

$$
\begin{aligned}
\operatorname{Var}\left[r_{1}(\tau)\right] & =\mathbb{E}\left[\left(r_{1}(\tau)-\hat{r}_{1}(\tau)\right)^{2}\right] \\
& =\frac{1}{M} \sum_{u=1}^{D} \sum_{x=1}^{D} \delta_{1 u, 1 x}(\tau) \doteq \frac{\delta_{1}(\tau)}{M} .
\end{aligned}
$$

In Fig. 6, we plot the solution for $\delta_{1}(\tau)$ computed numerically for the $(3,6,50)$ ensemble (a) and for the $(4,8,100)$ ensemble (b) for the following $\epsilon$ values: $0.45(\triangleleft), 0.46(\circ)$, and $0.47(\triangleright)$. The dashed lines with the $(*)$ marker correspond to $\delta_{1}(\tau)$ for $\epsilon=0.45$ and the same ensembles with twice the chain lengths, i.e., $L=100$ in (a) and $L=200$ in (b). As $\hat{r}_{1}(\tau)$ in Fig. 2, $\delta_{1}(\tau)$ presents an approximate a flat evolution during the steady state phase (as in the mean evolution case, small wiggles can be observed by magnification). Denote by $\delta_{1}^{*}$ the value of the variance during the steady state phase. Note also that $\delta_{1}^{*}$ is roughly constant with $\epsilon$ for the set of values considered. Indeed, this same effect has been noticed for all tested configurations of the $(l, r, L)$ ensemble and $\epsilon$ values sufficiently distant from the ensemble threshold $\epsilon_{(l, r, L)}$. In the following, we will use as representative value to $\delta_{1}^{*}$ the one computed at $\epsilon=\epsilon_{(l, r, L)}-0.04$. Obviously, further corrections on the scaling law that is proposed in this paper to estimate the $(l, r, L)$ performance can consider dropping this assumption and modeling $\delta_{1}^{*}$ as a function of $\epsilon$, or integrating the covariance evolution equations for each $\epsilon$ value. In Table III, we summarize the $\delta_{1}^{*}$ values computed for different configurations of the $(l, r, L)$ ensemble with $L=100$.

An important figure of merit to evaluate the finite-length scaling properties of $(l, r)$-regular LDPC code ensembles is the ratio of the expected number of degree-one check nodes at the critical point, $\hat{r}_{1}(*)$, to its standard deviation [16]. As

TABLE III

$\delta_{1}^{*}$ PARAMETER COMPUTED FOR DIFFERENT $(l, r, L)$ ENSEMBLES.

\begin{tabular}{|c|c|c|c|}
\hline 1 & $\mathrm{r}$ & $\delta_{1}^{*}$ & $\gamma / \sqrt{\delta_{1}^{*}}$ \\
\hline 3 & 6 & 0.67 & 5.12 \\
4 & 8 & 0.85 & 4.44 \\
5 & 10 & 0.91 & 4.23 \\
6 & 12 & 1.05 & 4.04 \\
4 & 12 & 0.64 & 5.1 \\
5 & 15 & 0.72 & 4.72 \\
4 & 6 & 0.91 & 4.28 \\
\hline
\end{tabular}

Covariance evolution differential equations:

$$
\frac{\partial \delta_{z, x}^{j, u}(\tau)}{\partial \tau}=f_{z, x}^{j, u}(\hat{\mathcal{G}}(\tau))-f_{j, u}(\hat{\mathcal{G}}(\tau)) f_{z, x}(\hat{\mathcal{G}}(\tau))+\left.\sum_{q=1}^{r} \sum_{m=1}^{D} \delta_{q, m}^{j, u}(\tau) \frac{\partial f_{z, x}(\mathcal{G}(\tau))}{\partial \mathcal{G}_{q, m}(\tau)}\right|_{\hat{\mathcal{G}}(\tau)}+\left.\delta_{z, x}^{q, m}(\tau) \frac{\partial f_{j, u}(\mathcal{G}(\tau))}{\partial \mathcal{G}_{q, m}(\tau)}\right|_{\hat{\mathcal{G}}(\tau)}
$$

for $(u, x) \in[D]^{2},(j, z) \in[r+1]^{2} \cdot \hat{\mathcal{G}}(\tau)$ is the expected graph evolution computed by solving (11). 
we show in Section $\mathrm{V}$, the same figure of merit, associated to the mean and variance of the $r_{1}(\tau)$ process during the critical phase, appears in the scaling law proposed for the $(l, r, L)$ ensemble:

$$
\frac{\hat{r}_{1}(*)}{\sqrt{\delta_{1}^{*} / M}} \approx \frac{\gamma \sqrt{M}\left(\epsilon_{(l, r, L)}-\epsilon\right)}{\sqrt{\delta_{1}^{*}}} .
$$

One of the main conclusions of this work is that the error probability of the $(l, r, L)$ decreases exponentially fast with (23). Thus, small differences in $\gamma / \sqrt{\delta_{1}^{*}}$ for different configurations might have a noticeable impact in the finite-length performance of the code. In Table III, we also include the ratio $\gamma / \sqrt{\delta_{1}^{*}}$ computed for the different $(l, r, L)$ ensembles $\left(\delta_{1}^{*}\right.$ is in the same table and $\gamma$ can be found in Table Ip. Note that, for fixed $l / r$ ratio, $\gamma / \sqrt{\delta_{1}^{*}}$ tends to decrease as we increase $l$, suggesting that once the threshold is sufficiently close to capacity, increasing the code density does not improve the finite-length performance of the code. For instance, this will be the case between the $(5,10,100)$ and $(6,12,100)$ ensembles.

\section{A. Process covariance at two time instants}

As we mentioned before, unlike for the uncoupled $(l, r)$ regular LDPC code ensemble, the error probability of the coupled ensemble is not determined by the behavior of the decoder at a particular "critical" point in time. As illustrated in Fig. 2, the decoder remains in a critical state during a period of time of duration $\Theta(L)$. Therefore, the error probability in the coupled case is given by the cumulative probability that the $r_{1}(\tau)$ process hits zero at some point during the critical phase. Recall that for the purpose of this paper we ignore errors which may happen in either the initial or the final phase, since they are very rare.

To compute the error probability during the steady state phase, we have to take into account the covariance of the $r_{1}(\tau)$ process over time:

$$
\phi_{1}(\zeta, \tau) \doteq \mathbb{E}\left[r_{1}(\zeta) r_{1}(\tau)\right]-\hat{r}_{1}(\zeta) \hat{r}_{1}(\tau),
$$

where $\tau$ and $\zeta$ are two distinct time instances. The expectation is defined over the joint probability distribution of the DD at times $\tau$ and $\zeta$, and we denote the corresponding p.d.f. by $p_{\mathcal{G}(\zeta), \mathcal{G}(\tau)}(g(\zeta), g(\tau))$. The quantity $\phi_{1}(\zeta, \tau)$ can in principle be computed analytically by a procedure similar to covariance evolution. However, while the number of coupled equations in the covariance evolution system in (19.B) is $(D \times(r+1))^{2}$, the number of coupled equations in the system that we would need to solve to be able to compute $\phi_{1}(\zeta, \tau)$ analytically is at least $(D \times(r+1))^{4}$ since we have to consider a minimum of two consecutive PD iterations. This approach is complex and computationally challenging.

Some thought shows that $\phi_{1}(\zeta, \tau)$ in 24) should be a function of $|\tau-\zeta|$ and that the decay of the correlation should be exponential in this time difference, i.e.,

$$
\phi_{1}(\zeta, \tau) \approx \frac{\delta_{1}^{*}}{M} \mathrm{e}^{-\theta|\tau-\zeta|},
$$

where $\theta$ is a parameter that depends on the $(l, r)$-regular LDPC ensemble and the coupling pattern of the SC-LDPC code. Simulations support the ansatz 25. For instance, in
Fig. 7. we plot (thin solid line) an estimation of $M \phi_{1}(\zeta, \tau)$ for the $(3,6,50)$ ensemble, $\epsilon=0.45$ and $\zeta=13$ computed by simulating 500 transmitted codewords with a code generated with $M=1000$ bits per position. Empirically we have observed that in order to accurately estimate the value of $\theta$ for a given $(l, r, L)$ ensemble, we only require a few hundred transmitted codewords. Why is it natural to consider the ansatz in 25]? In [33], in the context of the finite-length analysis of uncoupled ensembles, it was shown that in this case the correlation of BP messages decays exponentially in the graph distance. For us the natural equivalent of graph distance is the difference in decoding time.

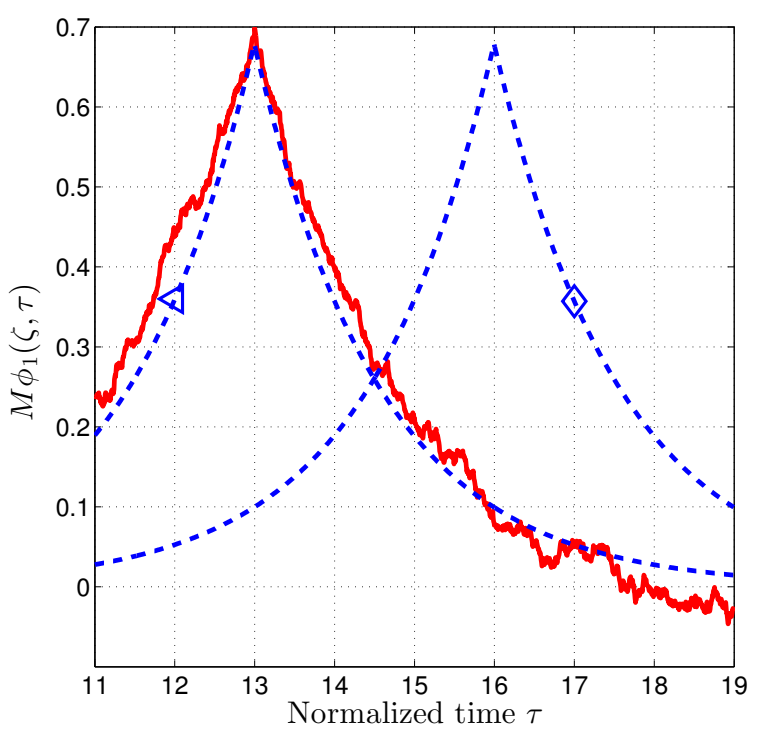

Fig. 7. $M \phi_{1}^{N}(\zeta, \tau)$ (dashed lines) computed for the $(3,6,50)$ ensemble and $N=200$ samples. We have fixed $\zeta$ to $13(\triangleleft)$ and $16(\diamond)$. For $\zeta=$ 13 , we have also included an estimation of $M \phi_{1}(\zeta, \tau)$ computed using 500 transmitted codewords for a code generated with $M=1000$ bits per position.

An alternative method to estimate $\theta$, that is based solely on the analytical expressions derived before to compute the mean and covariance of the residual graph along peeling decoding, is as follows. With no loss of generality, we assume $\tau>\zeta$. First, we express the correlation term in 24] in a more convenient way:

$$
\begin{aligned}
& \mathbb{E}\left[r_{1}(\zeta) r_{1}(\tau)\right] \\
& =\iint r_{1}(\zeta) r_{1}(\tau) p_{\mathcal{G}(\zeta), \mathcal{G}(\tau)}(g(\zeta), g(\tau)) \mathrm{d} g(\zeta) \mathrm{d} g(\tau) \\
& =\int r_{1}(\zeta) \mathbb{E}\left[r_{1}(\tau) \mid g(\zeta)\right] p_{\mathcal{G}(\zeta)}(g(\zeta)) \mathrm{d} g(\zeta),
\end{aligned}
$$

where the expectation inside the integral in 26) is taken with respect the conditional probability distribution

$$
p_{\mathcal{G}(\tau) \mid g(\zeta)}(g(\tau))
$$

i.e. the graph DD probability distribution at $\tau$ if the graph DD at $\zeta$ is fixed to $g(\zeta)$. Note that, for any DD $g(\zeta)$, we can easily evaluate $\mathbb{E}\left[r_{1}(\tau) \mid g(\zeta)\right]$ using the same equations derived to compute expected graph evolution in Section III and initial conditions given by $g(\zeta)$. Regarding the distribution 
$p_{\mathcal{G}(\zeta)}(g(\zeta))$ of the DD at time $\zeta$, all the $\mathcal{G}_{j, u}(\tau)$ terms in $\mathcal{G}(\tau)$, $u \in[D]$ and $j \in[r+1]$, are jointly Gaussian distributed with mean and covariance given as the solution to the system of differential equations in (12) and 19.B.

Therefore, the solution to the integral in 26) can be estimated by taking samples 8 from $\mathcal{G}(\zeta)$, where each sample is a possible DD at $\zeta$. For each sample, $\mathbb{E}\left[r_{1}(\tau) \mid g(\zeta)\right]$ can be obtained by numerical integration of the expected graph evolution equations in (12) using the sampled DD as initial conditions. Let $\mathcal{S}$ be the collection for $N$ samples taken of the graph DD at time $\zeta$. The integral in $(26)$ is approximated by a sum of the form

$$
\frac{1}{N} \sum_{g(\zeta) \in \mathcal{S}} r_{1}(\zeta) \mathbb{E}\left[r_{1}(\tau) \mid g(\zeta)\right]
$$

In the limit of $N \rightarrow \infty$

$$
\phi_{1}^{N}(\zeta, \tau) \doteq \frac{1}{N} \sum_{g(\zeta) \in \mathcal{S}} r_{1}(\zeta) \mathbb{E}\left[r_{1}(\tau) \mid g(\zeta)\right]-\hat{r}_{1}(\zeta) \hat{r}_{1}(\tau)
$$

converges to the true covariance $\phi_{1}(\zeta, \tau)$ [34]. It is important to remark that $\phi_{1}^{N}(\zeta, \tau)$ is a quantity obtained using exclusively the equations that predict the mean and variance graph evolution for the $(l, r, L)$ ensemble derived in Sections III and IV respectively.

In Fig. 7. we plot in dashed lines $M \phi_{1}^{N}(\zeta, \tau)$ computed for the $(3,6,50)$ ensemble at $\epsilon=0.45$ with $N=200$ samples. We have fixed $\zeta$ to $13(\triangleleft)$ and $16(\diamond)$. As predicted, the covariance decays exponentially with $|\tau-\zeta|$. In Table IV, we show the $\theta$ value computed for different $(l, r, L)$ ensembles at $\epsilon=\epsilon_{(l, r, L)}-0.04$. As we can observe, for fixed rate, the covariance decays slightly faster as we increase the check degree. A more detailed description of the effect of $\theta$ (and the rest of scaling parameters) in the finite-length performance is left to Section $\mathrm{V}$

TABLE IV

$\theta$ PARAMETER FOR DIFFERENT $(l, r, L)$ ENSEMBLES.

\begin{tabular}{|c|c|c|}
\hline 1 & $\mathrm{r}$ & $\theta$ \\
\hline 3 & 6 & 0.59 \\
4 & 8 & 0.61 \\
5 & 10 & 0.63 \\
4 & 12 & 0.84 \\
5 & 15 & 0.88 \\
4 & 6 & 0.51 \\
\hline
\end{tabular}

Finally, it is important to note that the decay of correlation of the $r_{1}(\tau)$ process, and hence the $\theta$ parameter in 25, does not only depend on the uncoupled $(l, r)$-regular LDPC code ensemble but also on the coupling pattern that we use to generate the coupled ensemble. In order to illustrate this dependence, consider a modification of the $(l, r, L)$ ensemble, denoted by $\mathcal{E}(l, r, L, w)$ where $w$ is a positive integer. For this ensemble, the coupling pattern as follows: for $u=1, \ldots, D$

\footnotetext{
${ }^{8}$ Sampling from $\mathcal{G}(\zeta)$ is straightforward since it is Gaussian distributed.
}

- If $u$ is odd, then each variable node at position $u$ is connected to a check node at position $u, u+w, u+$ $2 w, \ldots, u+w(l-1)$.

- If $u$ is even, then each variable node at position $u$ is connected to a check node at position $u, u+1, u+$ $2, \ldots, u+(l-1)$.

In words, we are "stretching" out the connections over a length that is $w$ times larger. In analogy to convolutional codes, we are increasing the "constraint length" of the code. Note that this ensemble has $D=L+w(l-1)$ positions and hence the design rate is smaller than the design rate of the $(l, r, L)$ ensemble. A representation of the $\mathcal{E}(3,6, L, 2)$ ensemble can be found in Fig. 8

What is the expected effect of this modification? As we increase $w$, we are further and further spreading out the connections in the spatial dimension and hence we expect the slower decay of the covariance of the process $r_{1}(\tau)$. Indeed, this effect can be observed in Fig. 9, where we show the covariance decay of the process $r_{1}(\tau)$ for the $(3,6,100)$ ensemble $(\triangleleft)$ and the $\mathcal{E}(3,6,100, w)$ ensemble with $w=2$ $(\triangleright)$ and $w=4(\square)$. For each ensemble we represent $M \phi_{1}(\zeta, \tau) / \delta_{1}^{*}$ so that all curves have a maximum equal to 1. The covariance has been estimated using 500 transmitted codewords (thin dashed lines) and using the alternative method summarized by equation (29) (solid lines), with $N=200$ samples. As expected, the covariance between $r_{1}(\tau)$ and $r_{1}(\zeta=29)$ is higher for larger $w$ values. The estimated $\theta$ values are, respectively, $\theta=0.59, \theta=0.28(w=2)$ and $\theta=0.17(w=4)$.

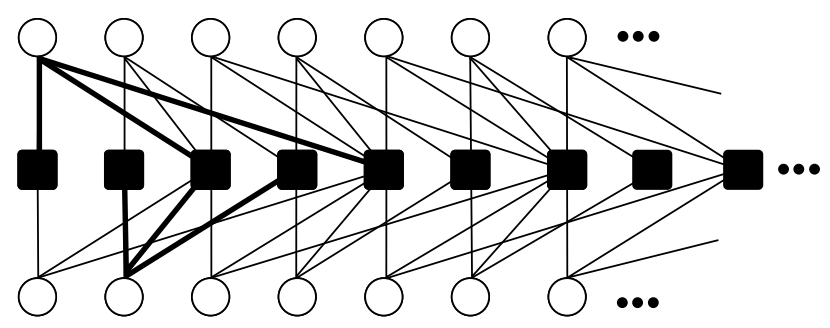

Fig. 8. A graphical representation of the $(3,6, L, w)$ ensemble for $w=2$. In thick lines, we illustrate the connections of a variable node placed at an odd position and a variable node at an even position.

At this point, we have all the ingredients we need to predict the survival probability of the process $r_{1}(\tau)$. Before concluding the present section, we finally want to emphasize that the triple $\left(\gamma, \delta_{1}^{*}, \theta\right)$ only depends on the uncoupled ensemble and the coupling pattern. The larger we choose $L$, the longer the process $r_{1}(\tau)$ remains in the steady state phase and thus the larger the error probability will be. However, the intrinsic statistical properties of the $r_{1}(\tau)$ during this phase process do not vary if we increase the chain length $L$. To illustrate this property, in Fig. 2 and Fig. 6 we have included results for both the $(3,6,50)$ and $(4,8,100)$ ensembles and the same ensembles with double chain lengths, $L=100$ and $L=200$ respectively. 


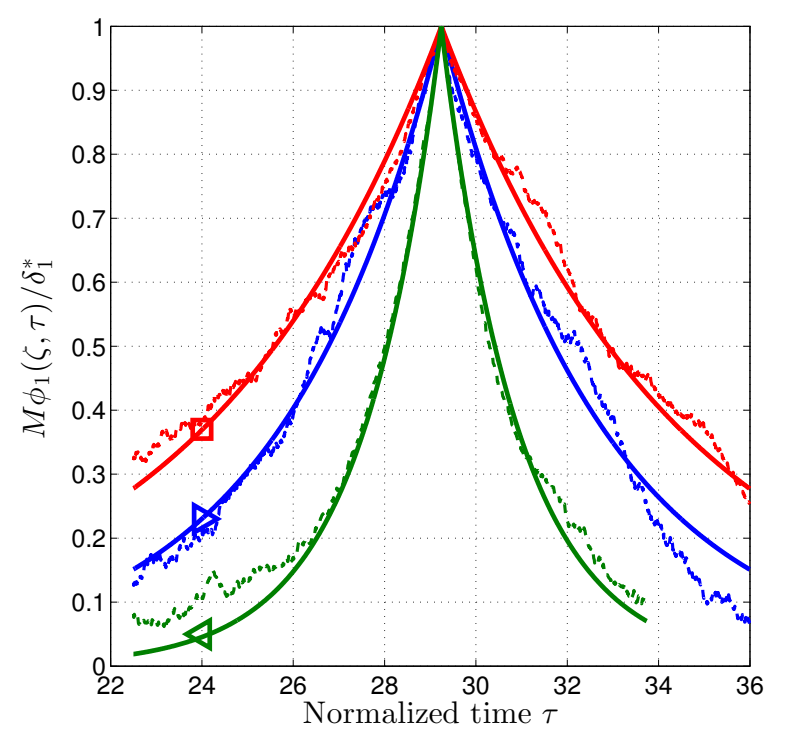

Fig. 9. $\phi_{1}^{N}(\zeta, \tau)$ with $N=200$ samples computed for the $(3,6,100)$ ensemble $(\triangleleft)$ and the $\mathcal{E}(3,6,100, w)$ ensemble with $w=2(\triangleright)$ and $w=$ $4(\square)$. We have fixed $\zeta$ to 29. In thin dashed lines, we also include the corresponding estimate computed using 500 transmitted codewords.

\section{PREDICTION OF THE ERROR PROBABILITY USING ORNSTEIN-UHLENBECK PROCESSES}

In previous sections, we have provided a statistical characterization of the process $r_{1}(\tau)$ for the $(l, r, L)$ ensemble when used for transmission over the BEC and decoded via PD. As proven in [16], $r_{1}(\tau)$ is a Markov process that converges (in the number $M$ of bits per position of the code) to a Gaussian process. Further, covariance evolution for the $(l, r, L)$ ensemble shows that $r_{1}(\tau)$ in the steady state phase is essentially a constant-mean and constant-variance process whose temporal covariance only depends on the time difference between the two time points considered. In other words, in the steady state phase, $r_{1}(\tau)$ is well modeled by a stationary Gaussian Markov process. Indeed, a stationary Gaussian Markov process $X(t)$ can only be one of the two following types [22], [35]:

a) If $t_{1}<t_{2}<\ldots<t_{n}, X\left(t_{1}\right), \ldots, X\left(t_{n}\right)$ are mutuallyindependent Gaussian random variables.

b) There exists a constant $\alpha$ such that if $t_{1}<t_{2}<\ldots<t_{n}$, then $X\left(t_{1}\right), \ldots, X\left(t_{n}\right)$ are jointly distributed by a multivariate Gaussian with common mean and variance and covariance function $\operatorname{CoVar}[X(t+T), X(t)] \propto \exp ^{-\alpha T}$,

where the latter type of Gaussian Markov process is called an Ornstein-Uhlenbeck (OU) process [22]. Therefore, under the Gaussian assumption for the distribution of $r_{1}(\tau)$, an OU process is the only type of stationary Gaussian Markov process that is compatible with the process $r_{1}(\tau)$. In the following we describe the properties and parameters of OU processes and link them with those already computed for $r_{1}(\tau)$ in previous sections.

\section{A. Ornstein-Uhlenbeck Processes}

An OU process is a stationary Gaussian Markov process evolving via the following stochastic equation [22]:

$$
X(t)=X_{0} \mathrm{e}^{-a t}+\sqrt{2 b} \mathrm{e}^{-a t} \int_{0}^{t} \omega(u) \mathrm{e}^{a u} \mathrm{~d} u,
$$

where $\omega(t)$ is a white noise with zero mean and unit variance, $a$ and $b$ are real positive constants and $X_{0}$ is the initial condition. Consider samples uniformly taken from $X(t)$ every $\Omega$ seconds, where $X_{i}$ is the $i$-th sample. The mean of $X_{i}$ is given by

$$
\mathbb{E}\left[X_{i}\right]=X_{0} \mathrm{e}^{-a i \Omega}=X_{0} g^{i}, \quad g \doteq \mathrm{e}^{-a \Omega} .
$$

Similarly, the covariance function is given by

$$
\mathbb{E}\left[\left(X_{i}-\mathbb{E}\left[X_{i}\right]\right)\left(X_{j}-\mathbb{E}\left[X_{j}\right]\right)\right]=\frac{b}{a}\left(g^{|i-j|}-g^{i+j}\right) .
$$

Therefore, for sufficiently large $i$,

$$
\begin{aligned}
& X_{i} \sim \mathcal{N}\left(0, \frac{b}{a}\right), \\
& \mathbb{E}\left[\left(X_{i}-\mathbb{E}\left[X_{i}\right]\right)\left(X_{j}-\mathbb{E}\left[X_{j}\right]\right)\right]=\frac{b}{a}\left(g^{|i-j|}\right) .
\end{aligned}
$$

If we recall the properties observed for the process $r_{1}(\tau)$ during the steady state phase:

$$
\begin{aligned}
& \mathbb{E}\left[r_{1}(\tau)\right]=\hat{r_{1}}(*)=\gamma\left(\epsilon_{(l, r, L)}-\epsilon\right), \\
& \left.\operatorname{Var}\left[r_{1}(\tau)\right]\right)=\frac{\delta_{1}^{*}}{M}, \\
& \mathbb{E}\left[r_{1}(\zeta) r_{1}(\tau)\right]-\mathbb{E}\left[r_{1}(\zeta)\right] \mathbb{E}\left[r_{1}(\tau)\right]=\frac{\delta_{1}^{*}}{M} \mathrm{e}^{-\theta|\tau-\zeta|},
\end{aligned}
$$

we can conclude that the process $r_{1}(\tau)-\hat{r}_{1}(*)$ can be identified as an OU process with parameters:

$$
a=\theta, \quad b=\frac{\delta_{1}^{*} \theta}{M},
$$

where we have taken $\Omega=M^{-1}$, i.e., the normalized time for a single PD iteration.

\section{B. First-passage time distribution}

The statistical distribution of the first-passage time (FPT) of an Ornstein-Uhlenbeck process, i.e., the first time at which an OU process is above a certain boundary $s$, is of interest in a variety of fields [23], [25]. Mathematically, if $X(t)$ is an OU process, the FPT for a boundary $s$ is defined as

$$
T_{s}=\inf _{t \geq 0}\{t: X(t) \geq s\} .
$$

Unlike the case of a Brownian motion, analytic expressions for the p.f.d. of $T_{s}, p_{T_{s}}(t)$, known to date are quite involved and for specific applications there is a need to perform numerical computations of the density [36]. As summarized by Alili, Patie and Pedersen in [37], three representations have been proposed for the first-passage time density of an OU-process through a constant boundary. The first expression is a series expansion involving the eigenvalues of a SturmLiouville boundary value problem associated with the Laplace transform of the FPT probability density function. The second one is an integral representation using its Fourier transform, 
and the third one is given in terms of a functional of a threedimensional Bessel bridge. Also, expressions for the moments of $T_{s}$ are only known in integral form [28], [38].

Assuming $X(\tau)=r_{1}(\tau)-\hat{r}_{1}(*)$ is a zero-mean OU process during the steady state phase, of length $\epsilon L-\tau^{*}$, to estimate the error probability we have to compute the cumulative probability

$$
P\left(0 \leq T_{s} \leq \epsilon L-\tau^{*}\right)=\int_{0}^{\epsilon L-\tau^{*}} p_{T_{s}}(t) d t
$$

for $s=\hat{r}_{1}(*)=\gamma\left(\epsilon_{(l, r, L)}-\epsilon\right)$. An estimate to 40) can be obtained by numerical integration using the analytic expressions of $p_{T_{s}}(t)$ commented above. While in principle this approach is valid, we are rather interested in a more informative expression that provides insights into the relation between the SC-LDPC structural parameters and the block error probability.

In [27], the authors show that $p_{T_{s}}(t)$ converges as $\frac{s}{b / a} \rightarrow \infty$ to an exponential distribution

$$
p_{T_{s}}(t) \sim \frac{1}{\mu_{0}} \mathrm{e}^{-t / \mu_{0}}
$$

where $\mu_{0}=\mathbb{E}\left[T_{s}\right]$ is the OU mean first-passage time from the zero initial state to the boundary $s$. In terms of the parameters of the $r_{1}(\tau)$ process in 38 we have

$$
\frac{s}{b / a}=\frac{\gamma M\left(\epsilon_{(l, r, L)}-\epsilon\right)}{\delta_{1}^{*}} \leq \frac{\gamma M \epsilon_{(l, r, L)}}{\delta_{1}^{*}} .
$$

and hence the exponential distribution in (41) is achieved in the limit $M \rightarrow \infty$. In addition, $\mu_{0}$ can be exactly computed using the following integral expression [28]:

$$
\mu_{0}(a, b, s)=\frac{\sqrt{2 \pi}}{a} \int_{0}^{\frac{s}{\sqrt{\frac{b}{a}}}} \Phi(z) \mathrm{e}^{\frac{1}{2} z^{2}} \mathrm{~d} z
$$

where $\Phi(z)$ is the c.d.f. of a Gaussian distribution with zero mean and unit variance, i.e.,

$$
\Phi(z)=\int_{-\infty}^{z} \frac{1}{\sqrt{2 \pi}} e^{-\frac{x^{2}}{2}} d x .
$$

In the limit $s \rightarrow \infty$ (the same limit for which the exponential distribution in 41) holds), the following upper bound to (43) becomes tight:

$$
\mu_{0}(a, b, s)<\frac{\sqrt{2 \pi}}{\sqrt{a b}} s \exp \left(\frac{s^{2}}{2 \frac{b}{a}}\right)
$$

Proof: Denote $C=\frac{s}{\sqrt{\frac{b}{a}}}$. Since $\Phi(z) \in[0,1]$,

$$
\begin{aligned}
\mu_{0}(a, b, s) & =\frac{\sqrt{2 \pi}}{a} \int_{0}^{C} \Phi(z) \mathrm{e}^{\frac{1}{2} z^{2}} \mathrm{~d} z \\
& <\frac{\sqrt{2 \pi}}{\theta} \int_{0}^{C} \mathrm{e}^{\frac{1}{2} z^{2}} \mathrm{~d} z \stackrel{(a)}{=} \frac{\sqrt{2 \pi}}{a} \sum_{n=0}^{\infty} \frac{2^{-n} C^{2 n+1}}{n !(2 n+1)}, \\
& =\frac{\sqrt{2 \pi}}{a} C \sum_{n=0}^{\infty} \frac{\left(C^{2} / 2\right)^{n}}{n !(2 n+1)}
\end{aligned}
$$

where the equality $(a)$ is obtained by using the series expansion $\exp (x)=\sum_{n} x^{n} / n$ ! Further, 46 can be upper bounded as follows:

$$
\begin{aligned}
& \frac{\sqrt{2 \pi}}{a} C \sum_{n=0}^{\infty} \frac{\left(C^{2} / 2\right)^{n}}{n !(2 n+1)} \\
& <\frac{\sqrt{2 \pi}}{a} C \sum_{n=0}^{\infty} \frac{\left(C^{2} / 2\right)^{n}}{n !} \\
& =\frac{\sqrt{2 \pi}}{a} C \exp \left(C^{2} / 2\right)=\frac{\sqrt{2 \pi}}{\sqrt{a b}} s \exp \left(\frac{s^{2}}{2 \frac{b}{a}}\right) .
\end{aligned}
$$

In our decoding scenario, we model the process $r_{1}(\tau)-$ $\hat{r}_{1}\left(\tau^{*}\right)$ as an OU process with parameters given in $(38)$. Hence, $\mu_{0}$ represents the average survival time once $r_{1}(\tau)$ has entered the steady state phase. Using (38), we obtain

$$
\mu_{0}=\frac{\sqrt{2 \pi}}{\theta} \int_{0}^{\frac{\gamma}{\sqrt{\delta_{1}^{*}}} \sqrt{M} \Delta_{\epsilon}} \Phi(z) \mathrm{e}^{\frac{1}{2} z^{2}} \mathrm{~d} z,
$$

where $\Delta_{\epsilon}=\left(\epsilon_{(l, r, L)}-\epsilon\right)$. Note that the integral in 48 diverges; $\mu_{0}$, the expected time at which the process $r_{1}(\tau)$ dies, grows exponentially fast with $M$ and $\Delta_{\epsilon}$. By (45), for large $M \Delta_{\epsilon}, \mu_{0}$ is tightly upper bounded by

$$
\mu_{0}<\frac{\sqrt{2 \pi}}{\theta} \frac{\gamma}{\sqrt{\delta_{1}^{*}}} \sqrt{M} \Delta_{\epsilon} \exp \left(M \frac{\gamma^{2} \Delta_{\epsilon}^{2}}{2 \delta_{1}^{*}}\right) .
$$

By taking a sufficiently large $M$, we can make $\mu_{0}>>\epsilon L$, which means that the length of the critical phase is very short compared to the time that, in average, we have to wait until $r_{1}(\tau)$ takes zero value. This results in a small error probability.

\section{Scaling law for the $(l, r, L)$ ensemble}

The first-passage time probability distribution in (41) along with (48) constitutes the necessary tools to estimate the decoding error probability. The steady state phase roughly lasts between $\tau^{*}=\tau^{*}(l, r, L, \epsilon)$ and $\epsilon L$. Recall also that $\tau^{*}$ is lower bounded by $\underline{\tau}$, computed in Section III-E from the DE solution of the uncoupled $(l, r)$-regular LDPC code ensemble. We estimate the $(l, r, L)$ ensemble average probability using the exponential distribution c.d.f., obtaining the following scaling law (SL):

$$
\begin{aligned}
& \mathbb{E}_{(l, r, L)}\left[P_{B}(l, r, L, M, \epsilon)\right] \\
& \approx 1-\exp \left(-\frac{\epsilon L-\tau^{*}}{\mu_{0}}\right) \\
& \leq 1-\exp \left(-\frac{\epsilon L-\underline{\tau}}{\mu_{0}}\right) \\
& =1-\exp \left(-\frac{\epsilon L-\underline{\tau}}{\frac{\sqrt{2 \pi}}{\theta} \int_{0}^{\frac{\gamma}{\sqrt{\delta_{1}^{*}}} \sqrt{M \Delta_{\epsilon}}} \Phi(z) \mathrm{e}^{\frac{1}{2} z^{2}} \mathrm{~d} z}\right),
\end{aligned}
$$

where, $\mathbb{E}_{(l, r, L)}\left[P_{B}(l, r, L, M, \epsilon)\right]$ represents the expected block error probability of the $(l, r, L)$ ensemble with $M$ bits per 
position averaged over all codes in the ensemble and all channel realizations and $\Phi(z)$ is given in (44).

Note that the expression in 50 depends on the ensemble parameters $L$ and $M$ and on the following scaling parameters:

1) gap to threshold $\Delta_{\epsilon}=\epsilon_{(l, r, L)}-\epsilon$

2) mean parameter $\gamma$ (see Table II)

3) variance parameter $\delta_{1}^{*}$ (see Table III)

4) rate of correlation decay $\theta$ (see Table IV)

In addition, there is a (slight) dependence on $\underline{\tau}$, see Table $\mathrm{I}$

In Fig. 10 we compare simulated block error probability curves (solid lines) along with the prediction using the expression in (50) (dashed lines) for the ensembles $(3,6,50)$ (a) and $(4,8,50)$ (b). The number $M$ of bits per position is:

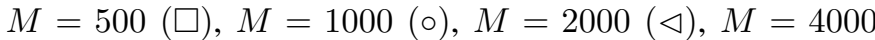
$(*)$ and $M=8000(\triangleright)$. For $M=8000$, we also include in dotted lines the estimated performance using the SL in 50 along with the upper bound to $\mu_{0}$ in (49).

First of all, we can see that there is a systematic "shift" between the actual error rate curves and the curves predicted via our scaling law. The estimate in (50) heavily relies on the assumption that the p.d.f. of the first-passage time of the $r_{1}(\tau)$ is distributed according to an exponential distribution. But this is an asymptotic result that only holds in the limit $M \rightarrow \infty$. In order to improve the estimate in (50), it is an interesting and challenging problem to drop the exponential distribution assumption and consider existing approaches to the p.d.f. of the first-passage time of an OU process for finite values of the boundary $s$ (controlled by $M$ in our problem) [37]. As we can observe in Fig. 10, for a few thousand bits per position, (50) provides an accurate estimate of the $(l, r, L)$ block error rate. At these lengths, SC-LDPC codes are prominent candidates for future communication standards such as optical communications [39] and wireless digital broadcasting [40]. Also, note that in all cases the slope of the error rate curves computed using (50) matches with the slope of the simulated error rates for the same $M$.

Hence, our first conclusion is that the $(l, r, L)$ performance can be accurately estimated if $M$ is sufficiently large. But our aim is to also show that the SL in (50) captures the right scaling between the ensemble block error rate and the different structural parameters, in particular the gap to threshold, $M$ and $L$. In this regard, the SL can be used to predict the performance improvement/degradation when a parameter is modified around a certain value. For instance, assume that for fixed $M$ and $L$, the waterfall performance of the $(l, r, L)$ ensemble has been estimated via Monte Carlo simulation. Now we can use the SL in (50) to predict the performance variation when the chain length and/or the number of bits per position are set to $L^{\prime}=c L$ and $M^{\prime}=k M$ respectively, $c, k \in \mathbb{R}^{+}$. These type of calculations are relevant from the practical point of view and the scaling law in 50 is a useful tool to perform quick estimates.

We will illustrate the problem in the low error rate regime. If we take a sufficiently large $M$, the error probability predicted by $(50)$ is small and we can use a first order Taylor expansion to simplify the expression:

$$
\mathbb{E}_{(l, r, L)}\left[P_{B}(l, r, L, M, \epsilon)\right] \approx \frac{\epsilon L-\underline{\tau}}{\mu_{0}} .
$$

In addition, in this regime the bound to $\mu_{0}$ in 49 ) is tight and hence,

$$
\mathbb{E}_{(l, r, L)}\left[P_{B}(l, r, L, M, \epsilon)\right] \approx \frac{\theta \sqrt{\delta_{1}^{*}}(\epsilon L-\underline{\tau})}{\sqrt{2 \pi} \gamma \sqrt{M} \Delta_{\epsilon}} \exp \left(-M \frac{\gamma^{2} \Delta_{\epsilon}^{2}}{2 \delta_{1}^{*}}\right)
$$

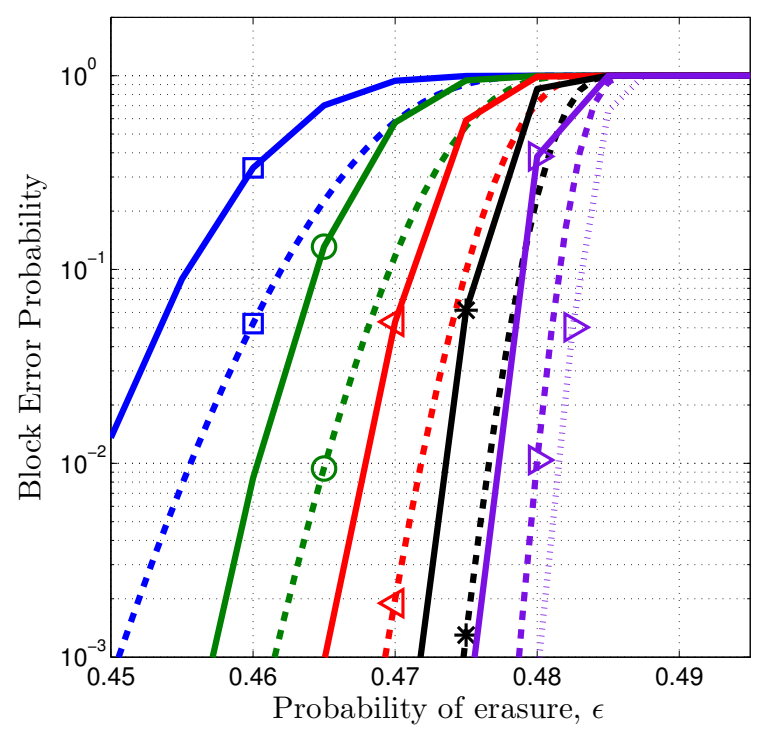

(a)

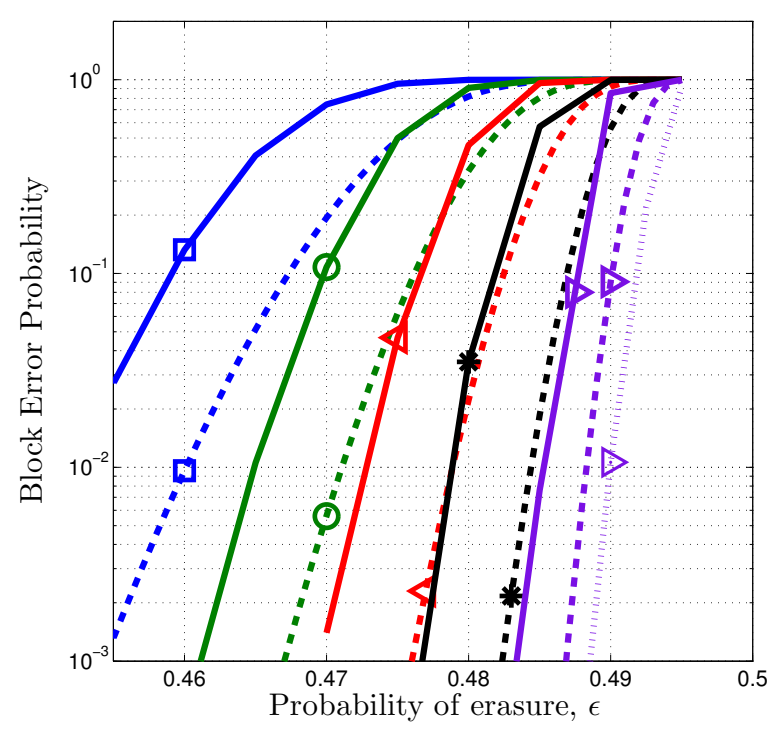

(b)

Fig. 10. Simulation error probability curves (solid lines) along with the estimated performance using the expression in [50] (dashed lines) for the ensemble $(3,6,50)$ (a) and $(4,8,50)$ (b) with $M=500(\square), M=1000$ (o), $M=2000(\triangleleft), M=4000(*)$ and $M=8000(\triangleright)$. For $M=8000$, we also include in dotted lines the estimated performance using the SL in [50) along with the upper bound to $\mu_{0}$ in 49 . 


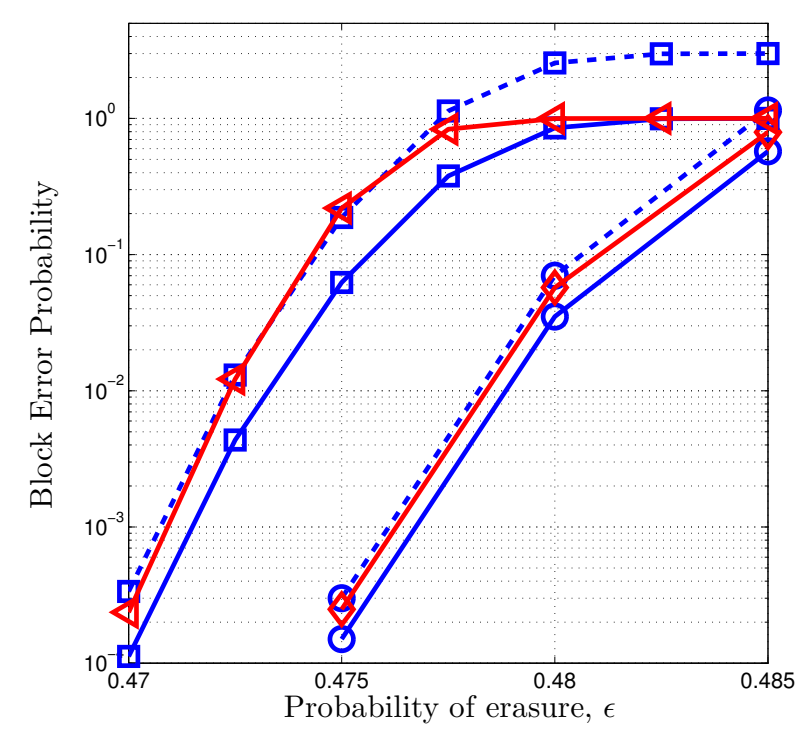

Fig. 11. Simulated error probability curves (solid lines) for the ensembles $(3,6,50)(\square),(3,6,150)(\triangleleft),(4,8,50)(\circ)$ and $(4,8,100)(\diamond)$ with $M=4000$ bits. In dashed lines, we represent the estimated error probability for the ensembles $(3,6,150)$ and $(4,8,100)$ computed by multiplying the simulated error rates for the ensembles $(3,6,50)$ and $(4,8,50)$ by three and two respectively.

Note that this expression indicates that the error probability scales linearly with the chain length. In Fig. 11, we represent the simulated error probability curves (solid lines) for the ensembles $(3,6,50)(\square),(3,6,150)(\triangleleft),(4,8,50)$ (०), $(4,8,100)(\diamond)$ with $M=4000$ bits. In dashed lines, we represent the estimated error probability for the ensembles $(3,6,150)$ and $(4,8,100)$ computed by multiplying the simulated error rates for the ensembles $(3,6,50)$ and $(4,8,50)$ by three and two respectively. Observe that, when the error probability is small enough the predicted performance matches with the simulated one for $L=150$ and $L=100$, indicating that the scaling in 51 w.r.t $L$ is essentially correct.

Now we test whether the scaling with respect to $M$ predicted by (52) is also accurate. Consider the $(l, r, L)$ ensemble with $M$ bits per position. Assume that we numerically find $M_{\mathrm{SL}}<M$ such that the simulated performance for $M$ bits per position and the one predicted by $(50)$ with $M_{\mathrm{SL}}$ match. Using the SL, we can now estimate how much the performance is improved when the number of bits per position is modified from $M$ to $k M$, for some $k>0$. Using the simplified expression in 52 for the low error rate regime, a simple calculation shows that

$$
\begin{aligned}
& \mathbb{E}_{(l, r, L)}\left[P_{B}(l, r, L, k M, \epsilon)\right] \\
& \approx \frac{\mathbb{E}_{(l, r, L)}\left[P_{B}(l, r, L, M, \epsilon)\right]}{\sqrt{k}} \exp \left(-M_{\mathrm{SL}} \frac{\gamma^{2}(k-1) \Delta_{\epsilon}^{2}}{2 \delta_{1}^{*}}\right) .
\end{aligned}
$$

The accuracy of the estimate in 53 in the low error rate regime is demonstrated in Fig. 12. In Fig. 12,a), we represent the simulated performance for the $(3,6,50)$ ensemble with $M=1000$ bits $(\square)$, solid line. The curve obtained is well approximated by 50 with $M_{\mathrm{SL}}=700$ bits (dotted line). Using this value, the dashed line with $(\triangleright)$ marker represents the estimated performance for the $(3,6,50)$ ensemble with $M=4000$ bits using (52), whereas the actual simulated performance for this case is plotted with solid line and (o) marker. As we can observe, the prediction is indeed accurate for small error rates. In Fig. 12 b), we show a similar example using the $(4,8,50)$ ensemble. The solid curve with ( $\square$ ) marker represents the simulated performance for the case $M=2000$ bits. This curve is well approximated by 50, with $M_{\mathrm{SL}}=1100$ bits and this value is used to compute the estimated performance for $M=4000$ bits, $(\triangleright)$ maker with dashed line. The actual $(4,8,50)$ performance is plotted with ( $\circ$ ) maker. In all cases, the simulated performance curves have been obtained after $10^{5}$ transmitted codewords. The values for $\gamma$ and $\delta_{1}^{*}$ in 52 are given for each ensemble in Table 1 and Table III.

The above results show that the SL proposed is consistent with the scaling behavior of the code. In [15], the performance of a class of SC-LDPC codes constructed from protographs was tested for a wide range of scaling functions $L=f(M)$. It was observed that the ensemble presented a vanishing error probability in the limit $M \rightarrow \infty$ for $\epsilon$ values very close to the MAP threshold of the uncoupled ensemble even though when $L$ grows much faster than $M$. In particular, we conjectured that this effect is lost only when the chain length $L$ grows at least exponentially fast with $M$. The scaling law for the $(l, r, L)$ ensemble in 50 is consistent with such a conjecture. Indeed, if $L=f(M)$ and $M$ is large enough then the SL in (50) takes the form:

$$
1-\exp \left(-\frac{\epsilon f(M)-\tau^{*}}{\frac{\sqrt{2 \pi}}{\theta} \frac{\gamma}{\sqrt{\delta_{1}^{*}}} \sqrt{M} \Delta_{\epsilon} \exp \left(\frac{\gamma^{2} M \Delta_{\epsilon}^{2}}{2 \delta_{1}^{*}}\right)}\right)
$$

and, hence, we can easily find a pair of real positive constants for which a scaling of the form $L=a \exp (b M)$ makes (54) tend to one in the limit $M \rightarrow \infty$. This result indicates that the wave-like decoding phenomenon, which allows achieving near-capacity thresholds, is very robust.

\section{Finite-length performance and scaling parameters}

Four scaling parameters appear in the scaling law proposed in (50), as well as in the low-error rate approximation in (52). In this regard, let us conclude this paper by briefly discussing the sensitivity of the SC-LDPC finite-length performance with respect to each one of them:

1) Mean parameter $\gamma$ and variance parameter $\delta_{1}^{*}$. First of all, note that these two parameters only appear in the SL by means of the ratio $\alpha \doteq \gamma / \sqrt{\delta_{1}^{*}}$. By (52), note the performance is very sensitive w.r.t. to any change in $\alpha$. A simple calculation shows that

$$
\frac{\partial \mathbb{E}_{(l, r, L)}\left[P_{B}(l, r, L, M, \epsilon)\right]}{\partial \alpha} \sim \mathcal{O}\left(-\mathrm{e}^{-\alpha^{2} \Delta_{\epsilon} M}\right),
$$

and thus a small increase in $\alpha$ can lead to a significant decay in the error rate. On one hand, we have shown how $\gamma$ is directly proportional to the speed of the wave 


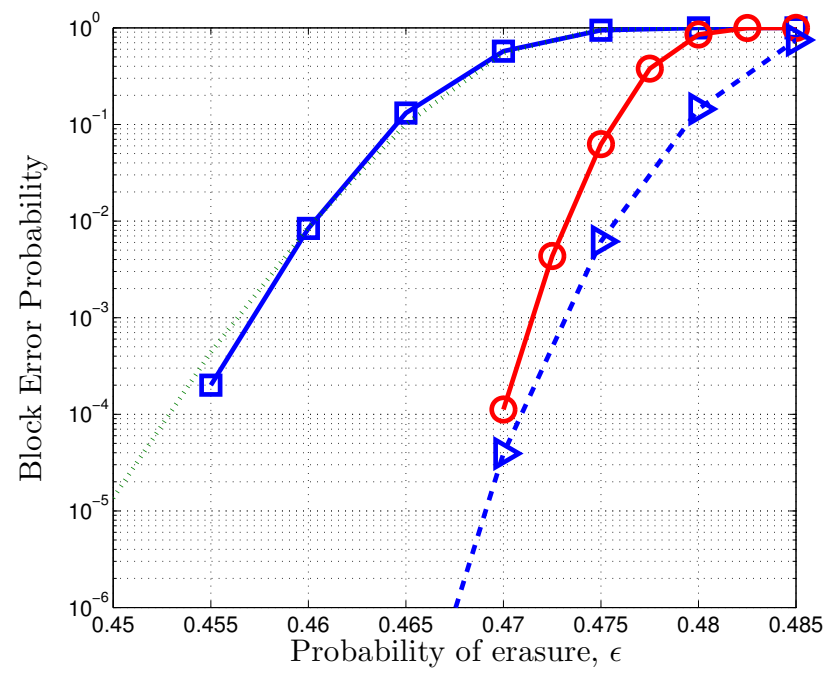

(a)

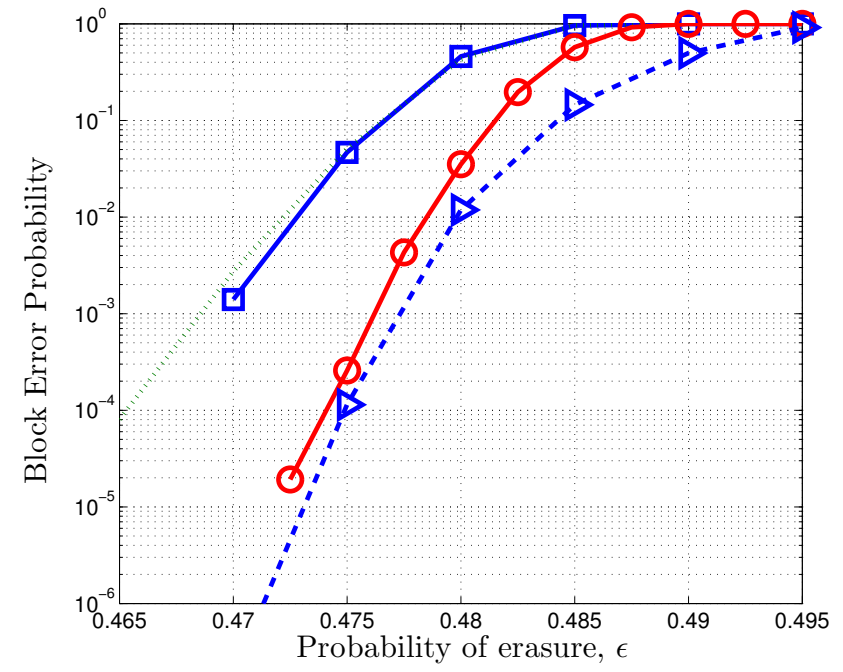

(b)

Fig. 12. In Fig. 12 we represent the simulated performance for the $(3,6,50)$ ensemble with $M=1000$ bits $(\square)($ a) and the $(4,8,50)$ ensemble with $M=2000$ bits ( $\square$ (b). In both cases, the dashed lines with $(\triangleright)$ marker represents the estimated performance using 52 for $M=4000$ bits. The actual $M=4000$ performance computed by simulation for each case is given by the solid line with (o) maker.

under BP message-passing decoding. From the design perspective, it has been shown how this speed can be improved by optimizing the ensemble degree profile [31]. On the other hand, the design of the SC-LDPC code ensemble to reduce the variance parameter $\delta_{1}^{*}$ is still a challenging open question since no closed-form expressions to compute $\delta_{1}^{*}$ (or bound it) have been proposed yet.

2) SC-LDPC code ensemble threshold. As in the case of $\alpha$, the finite-length performance is quite sensitive to $\Delta_{\epsilon}$. Since it is fairly easy to design SC-LDPC code ensembles with capacity-approaching thresholds [4], [9], the main question here is to determine whether the optimization of the SC-LDPC code ensemble to achieve thresholds arbitrarily close to capacity is compatible with the optimization of the rest of parameters that appear in the scaling law.

3) $\theta$ parameter. As described in Section IV-A $\theta$ is related to the covariance decay of the $r_{1}(\tau)$ process along the time and can be controlled by the coupling pattern. Intuitively, the more we spread the connections of variable nodes along different positions, the smaller $\theta$ value we can observe. However, it is not a trivial problem how to optimize this parameter at fixed coding rate (recall the coupling pattern also determines the boundary conditions, and thus, the coding rate loss incurred). In addition, upon optimization we might not observe a significant improvement in the finite-length performance since by (52) it only scales linearly with $\theta$.

\section{Vi. CONClusions AND Future Work}

We have analyzed the decoding of finite-length SC-LDPC codes over the BEC using the peeling decoder. By extending the methodology applied in [16] to study finite-length LDPC code ensembles, we have determined and solved covariance evolution, i.e., the system of differential equations that encodes the first two statistical moments of the evolution of the residual graph. We have shown that the statistical evolution of the normalized number of degree-one check nodes along the peeling decoding process, i.e., the $r_{1}(\tau)$ process, is of a completely different nature compared to the case of the uncoupled $(l, r$,$) -regular LDPC code ensemble. In the coupled ensemble,$ decoding failures more or less happen uniformly throughout the whole decoding process (with the exception of the very beginning and the very end of the process). Furthermore, the survival probability of the process $r_{1}(\tau)$ can be estimated by assuming that the $r_{1}(\tau)$-process is in fact a properly chosen Ornstein-Uhlenbeck process. The scaling law derived via this assumption contains four parameters: the ensemble threshold, the mean parameter $\gamma$, the covariance parameter $\delta_{1}^{*}$ and the correlation parameter $\theta$. The threshold can be determined through standard DE techniques. We derived the remaining parameters, namely $\left(\gamma, \delta_{1}^{*}, \theta\right)$, via covariance evolution.

The closed-form SL proposed in this paper provides code designers with some insight into on the main scaling behavior between the error rate and the different code parameters. The scaling law gives a good approximation to the actual performance (determined via simulations) and, furthermore, it can be used to accurately predict how the performance changes if we modify some base parameters, such as $L$ or $M$.

Several important practical questions remain open. First of all, it would be interesting to determine whether there exist alternative methods to evaluate the triple $\left(\gamma, \delta_{1}^{*}, \theta\right)$ efficiently. As we mentioned in the paper, at least the parameter $\gamma$ is directly related to the decoding speed, and this decoding speed in term can be computed via basic quantities that appear in density evolution. If it was able to derived closed form expressions for these parameters then in principle this would open the 
door to perform an optimization of the code parameters. Also, what are the trade-offs between the parameters $\left(\gamma, \delta_{1}^{*}, \theta\right)$ ? As we have discussed, we would like $\gamma$ to be large and $\theta$ and $\delta_{1}^{*}$ to be small. But most likely, these are conflicting goals.

Finally, we have ignored some relevant aspects. Most importantly we ignored the effect of the termination on the error probability, the effect of a windowed-decoding algorithm [10], or the effect of the exact code structure. All of these are likely going to play a role in practical applications and hence have to be considered. In particular, we should address the analysis of protograph-based SC-LDPC codes [4], [18] and spatiallycoupled structures based on generalized LDPC codes [41] or non-binary LDPC codes [42].

\section{REFERENCES}

[1] S. Kudekar, T. Richardson, and R. Urbanke, "Spatially Coupled Ensembles Universally Achieve Capacity under Belief Propagation," IEEE Transactions on Information Theory, vol. 59, no. 12, pp. 7761 - 7813, Dec. 2013.

[2] A. Jimenez Felstrom and K. Zigangirov, "Time-varying periodic convolutional codes with low-density parity-check matrix," IEEE Transactions on Information Theory, vol. 45, no. 6, pp. 2181 -2191, Sept. 1999.

[3] D. Costello, L. Dolecek, T. Fuja, J. Kliewer, D. Mitchell, and R. Smarandache, "Spatially coupled sparse codes on graphs: theory and practice," IEEE Communications Magazine, vol. 52, no. 7, pp. 168-176, July 2014.

[4] M. Lentmaier, A. Sridharan, D. J. Costello, Jr, and K. Zigangirov, "Iterative decoding threshold analysis for LDPC convolutional codes," IEEE Transactions on Information Theory, vol. 56, no. 10, pp. 5274 -5289 , Oct. 2010

[5] S. Kudekar, T. Richardson, and R. Urbanke, "Threshold saturation via spatial coupling: Why convolutional LDPC ensembles perform so well over the BEC," IEEE Transactions on Information Theory, vol. 57, no. 2, pp. 803-834, Feb. 2011.

[6] D. Mitchell, A. Pusane, M. Lentmaier, and D. J. Costello, Jr, "Exact free distance and trapping set growth rates for LDPC convolutional codes," in Proc. IEEE International Symposium on Information Theory (ISIT), St. Petersburg, Russia, Aug. 2011, pp. $1096-1100$.

[7] A. Sridharan, D. Truhachev, M. Lentmaier, D. Costello, and K. Zigangirov, "Distance Bounds for an Ensemble of LDPC Convolutional Codes," IEEE Transactions on Information Theory, vol. 53, no. 12, pp. 4537-4555, Dec 2007.

[8] J. Thorpe, "Low density parity check (LDPC) codes constructed from protographs," JPL IPN Progress Report 42-154, Tech. Rep., 2003.

[9] M. Lentmaier, D. Mitchell, G. Fettweis, and D. J. Costello, Jr, "Asymptotically regular LDPC codes with linear distance growth and thresholds close to capacity," in Proc. Information Theory and Applications (ITA) Workshop, San Diego, CA, Feb. 2010.

[10] A. Iyengar, M. Papaleo, P. Siegel, J. Wolf, A. Vanelli-Coralli, and G. Corazza, "Windowed decoding of protograph-based LDPC convolutional codes over erasure channels," IEEE Transactions on Information Theory, vol. 58, no. 4, pp. 2303 - 2320, April 2011.

[11] R. Tanner, D. Sridhara, A. Sridharan, T. Fuja, and D. J. Costello, Jr, "LDPC block and convolutional codes based on circulant matrices," IEEE Transactions on Information Theory, vol. 50, no. 12, pp. 2966 - 2984, Dec. 2004.

[12] N. Hassan, M. Lentmaier, and G. Fettweis, "Comparison of LDPC block and LDPC convolutional codes based on their decoding latency," in Proc. IEEE International Symposium on Turbo Codes and Iterative Information Processing (ISTC), Gothenburg, Sweden, Aug 2012, pp. 225-229.

[13] A. Pusane, A. Feltstrom, A. Sridharan, M. Lentmaier, K. Zigangirov, and D. J. Costello, Jr, "Implementation aspects of LDPC convolutional codes," IEEE Transactions on Communications, vol. 56, no. 7, pp. 1060 -1069 , July 2008

[14] I. Bocharova, B. Kudryashov, and R. Johannesson, "LDPC convolutional codes versus QC LDPC block codes in communication standard scenarios," in Proc. IEEE International Symposium on Information Theory (ISIT), Hawaii, USA, June 2014, pp. 2774-2778.

[15] P. M. Olmos and R. Urbanke, "Scaling behavior of Convolutional LDPC ensembles over the BEC," in Proc. IEEE International Symposium on Information Theory (ISIT), St. Petersburg, Russia, Aug. 2011, pp. 1816 -1820 .
[16] A. Amraoui, A. Montanari, T. Richardson, and R. Urbanke, "Finitelength scaling for iteratively decoded LDPC ensembles," IEEE Transactions on Information Theory, vol. 55, no. 2, pp. 473 -498, Feb. 2009.

[17] T. Nozaki, K. Kasai, and K. Sakaniwa, "Analytical Solution of Covariance Evolution for Irregular LDPC Codes," IEEE Transactions on Information Theory, vol. 58, no. 7, pp. 4770-4780, July 2012.

[18] M. Stinner and P. Olmos, "Analyzing finite-length protograph-based spatially coupled LDPC codes," in Proc. IEEE International Symposium on Information Theory (ISIT), Hawaii, USA, June 2014, pp. 891-895.

[19] A. Amraoui, R. Urbanke, and A. Montanari, "Finite-length scaling of irregular LDPC code ensembles," in Proc. IEEE Information Theory Workshop (ITW), Rotorua, New Zealand, Aug. 2005.

[20] M. Luby, M. Mitzenmacher, M. Shokrollahi, and D. Spielman, "Efficient erasure correcting codes," IEEE Transactions on Information Theory, vol. 47 , no. 2 , pp. $569-584$, Feb. 2001.

[21] T. J. Richardson and R. Urbanke, Modern Coding Theory. Cambridge University Press, Mar. 2008.

[22] I. Karatzas and S. E. Shreve, Brownian Motion and Stochastic Calculus (Graduate Texts in Mathematics), 2nd ed. Springer, Aug. 1991.

[23] J. M. Beaulieu, D.-C. Jhwueng, C. Boettiger, and B. C. O'Meara, "Modeling Stabilizing Selection: Expanding the Ornstein-Uhlenbeck model of Adaptive Evolution," Evolution, vol. 66, no. 8, pp. 2369-2383, August 2012.

[24] O. Aalen and H. K. Gjessing, "Survival models based on the OrnsteinUhlenbeck Process," Lifetime Data Analysis, vol. 10, no. 4, pp. 407-423, 2004.

[25] P. Collin-Dufresne and R. S. Goldstein, "Do Credit Spreads Reflect Stationary Leverage Ratios?" The Journal of Finance, vol. 56, no. 5, pp. pp. 1929-1957, 2001.

[26] O. Onalan, "The Ornstein-Uhlenbeck Processes driven by Lévy process and Application to Finance," in Electronic Engineering and Computing Technology, ser. Lecture Notes in Electrical Engineering. Springer Netherlands, 2010, vol. 60, pp. 443-453.

[27] A. G. Nobile, L. M. Ricciardi, and L. Sacerdote, "Exponential trends of Ornstein-Uhlenbeck First-Passage-time densities," Journal of Applied Probability, vol. 22, no. 2, pp. 360-369, 1985.

[28] M. U. Thomas, "Some Mean First-Passage Time Approximations for the Ornstein-Uhlenbeck process," Journal of Applied Probability, vol. 12, no. 3, pp. 600-604, 1975 .

[29] G. Ehrhardt, S. Majumdar, and A. Bray, "Persistence exponents and the statistics of crossings and occupation times for gaussian stationary processes," Phys Rev E Stat Nonlin Soft Matter Phys, vol. 69, no. 1, 2004.

[30] N. C. Wormald, "Differential equations for random processes and random graphs," Annals of Applied Probability, vol. 5, no. 4, pp. 1217 $1235,1995$.

[31] V. Aref, L. Schmalen, and S. ten Brink, "On the Convergence Speed of Spatially Coupled LDPC Ensembles," Submitted to IEEE Transactions on Information Theory, 2013. [Online]. Available: http://arxiv.org/abs/1307.3780

[32] F. Caltagirone, S. Franz, R. Morris, and L. Zdeborov, "Dynamics and termination cost of spatially coupled mean-field models," CoRR, vol. abs/1310.2121, 2013. [Online]. Available: http://arxiv.org/abs/1310.2121

[33] S. Kudekar and N. Macris, "Decay of Correlations for Sparse Graph Error Correcting Codes," SIAM Journal on Discrete Mathematics, vol. 25 , no. 2, pp. 956-988, 2011

[34] K. P. Murphy, Machine learning: a probabilistic perspective. MIT press, 2012.

[35] J. L. Doob, The Brownian Movement and Stochastic Equations, ser. Second Series. Annals of Mathematics, 1942, vol. 43, no. 2.

[36] D. T. Gillespie, "Exact numerical simulation of the Ornstein-Uhlenbeck process and its integral," Physical review. E, Statistical physics, plasmas, fluids, and related interdisciplinary topics, vol. 54, no. 2, pp. 2084-2091, 1996.

[37] L. Alili, P. Patie, and J. Pedersen, "Representations of first hitting time density of an ornstein-uhlenbeck process," Stochastic Models, vol. 21, no. 4, pp. 967-980, 2005.

[38] L. M. Ricciardi and S. Sato, "First-Passage-Time Density and Moments of the Ornstein-Uhlenbeck Process," Journal of Applied Probability, vol. 25 , no. 1, pp. 43-57, 1988.

[39] F. Chang, K. Onohara, and T. Mizuochi, "Forward error correction for $100 \mathrm{G}$ transport networks," IEEE Communications Magazine, vol. 48, no. 3, 2010.

[40] “Digital Video Broadcasting-t2 standard, EN 302755 v.1.3.1." [Online]. Available: http://www.dvb.org/technology/dvbt2/index.xml 
[41] D. Mitchell, M. Lentmaier, and J. Costello, D.J., "On the minimum distance of generalized spatially coupled LDPC codes," in Proc. IEEE International Symposium on Information Theory (ISIT), Istanbul, Turkey, July 2013, pp. 1874-1878.

[42] K. Huang, D. Mitchell, L. Wei, X. Ma, and J. Costello, D.J., "Performance comparison of non-binary LDPC block and spatially coupled codes," in Proc. IEEE International Symposium on Information Theory (ISIT), Hawaii, USA, June 2014, pp. 876-880.

\section{APPENDIX A}

\section{EXPECTED EVOLUTION IN ONE ITERATION OF THE PD}

Here we show how to compute the expected evolution of the $(l, r, L)$ residual graph DD in one PD iteration, namely

$$
\begin{aligned}
& \mathbb{E}\left[R_{j, u}(\tau+1 / M)-R_{j, u}(\tau) \mid \mathcal{G}(\tau)\right], \\
& \mathbb{E}\left[V_{u}(\tau+1 / M)-V_{u}(\tau) \mid \mathcal{G}(\tau)\right],
\end{aligned}
$$

for $u \in[D]$ and $j \in[r]$. To keep the notation uncluttered, we omit the conditionality on $\mathcal{G}(\tau)$ and we denote

$$
\begin{aligned}
\Delta R_{j, u}(\tau) & =R_{j, u}(\tau+1 / M)-R_{j, u}(\tau), \\
\Delta V_{u}(\tau) & =V_{u}(\tau+1 / M)-V_{u}(\tau) .
\end{aligned}
$$

Let $\operatorname{pos}(\tau)$ be the position at which we remove a degree-one check node at time $\tau$. The actual check node that is removed is chosen uniformly at random from all degree-one check nodes at this position. The resulting probability distribution $P(\operatorname{pos}(\tau)=u)$ is described by $p_{u}$ in $(15)$.

Assume that $\operatorname{pos}(\tau)=m$. Given the properties of the $(l, r, L)$ ensemble described in Section II , it follows that the variable connected to this degree-one check node is placed at position $u$ with probability

$$
\lambda_{m, u}(\tau)=\frac{V_{u}(\tau)}{\sum_{i=m-(l-1)}^{m} V_{i}(\tau)}
$$

if $u \in\{m-(l-1), \ldots, m\}$ and zero otherwise. By extension, when a degree-one check node from position $m$ and the variable connected to it are removed, then with probability

$$
\xi_{m, u}(\tau)=\sum_{i=u-(l-1)}^{u} \lambda_{m, i}(\tau)
$$

one of the $l$ removed edges is connected to a check node at position $u, u \in[D]$. Note that $\xi_{u, u}(\tau)=1 \forall u$.

Given these definitions we can compute the expected evolution of the DD conditioned to the case for which the degree-one check node is removed from position $m$, i.e., $\operatorname{pos}(\tau)=m$. At position $m$, the variation in the DD is simple: $\mathbb{E}\left[\Delta R_{j, m}(\tau) \mid \operatorname{pos}(\tau)=m\right]$ is equal to -1 for $j=1$ and zero otherwise. At any other position $u \neq m$, an edge is removed from position $u$ with probability $\xi_{m, u}(\tau)$. The edge removed is connected to a check node of degree $j$ with probability

$$
\frac{R_{j, u}(\tau)}{\sum_{q=1}^{r} R_{q, u}(\tau)},
$$

and, if this happens, $j$ edges of right degree $j$ are removed from the graph at position $u$. Also, $j-1$ edges of right degree $j-1$ are created at the same position. Hence, the expected graph evolution at position $u \neq m$ is

$\mathbb{E}\left[\Delta R_{j, u}(\tau) \mid \operatorname{pos}(\tau)=m\right]=j \xi_{m, u}(\tau) \frac{R_{j+1, u}(\tau)-R_{j, u}(\tau)}{\sum_{q=1}^{r} R_{q, u}(\tau)}$, where for $j=r, R_{j+1, u}(\tau)=0$. Under the same assumption, $\operatorname{pos}(\tau)=m$, a variable node is removed from position $u$ with probability $\lambda_{m, u}(\tau)$. Therefore,

$$
\mathbb{E}\left[\Delta V_{u}(\tau) \mid \operatorname{pos}(\tau)=m\right]=-\lambda_{m, u}(\tau),
$$

and this holds for $u \in[D]$, including the case $u=m$.

Finally, the expected graph evolution after one iteration of the PD can be computed as follows. For $2 \leq j \leq(r-1)$ we get,

$$
\begin{aligned}
& \mathbb{E}\left[\Delta R_{j, u}(\tau)\right] \\
& =j \sum_{\substack{m=1 \\
m \neq u}}^{D} \xi_{m, u}(\tau) \frac{R_{j+1, u}(\tau)-R_{j, u}(\tau)}{\sum_{q=1}^{r} R_{q, u}(\tau)} p_{m}(\tau) \\
& =j\left(\frac{R_{j+1, u}(\tau)-R_{j, u}(\tau)}{\sum_{q=1}^{r} R_{q, u}(\tau)}\right)\left(\boldsymbol{p}^{T} \boldsymbol{\xi}_{u}-p_{u}(\tau)\right),
\end{aligned}
$$

where $\boldsymbol{p} \doteq\left[p_{1}(\tau) \ldots p_{D}(\tau)\right]$ and $\boldsymbol{\xi}_{u}=\left[\xi_{1, u}, \xi_{2, u}, \ldots, \xi_{D, u}\right]$ are $D$-length vectors. For the case $j=1$, we also have to take into account the degree-one check node removed from the graph, which belongs to position $u$ with probability $p_{u}(\tau)$. Thus,

$$
\begin{aligned}
& \mathbb{E}\left[\Delta R_{1, u}(\tau)\right]= \\
& =-p_{u}(\tau)+\left(\frac{R_{2, u}(\tau)-R_{1, u}(\tau)}{\sum_{q=1}^{r} R_{q, u}(\tau)}\right)\left(\boldsymbol{p}^{T} \boldsymbol{\xi}_{u}-p_{u}(\tau)\right) .
\end{aligned}
$$

On the variable side we obtain

$$
\mathbb{E}\left[\Delta V_{u}(\tau)\right]=-\sum_{m=1}^{D} \lambda_{m, u}(\tau) p_{m}(\tau)
$$

\section{APPENDIX B}

\section{COVARIANCE EVOLUTION IN ONE ITERATION OF THE PD}

We are now interested in computing the second order moments for the $(l, r, L)$ ensemble residual DD transition at one PD iteration:

$$
\begin{aligned}
& \mathbb{E}\left[\Delta R_{j, u}(\tau) \Delta R_{z, x}(\tau) \mid \mathcal{G}(\tau)\right], \\
& \mathbb{E}\left[\Delta V_{u}(\tau) \Delta V_{x}(\tau) \mid \mathcal{G}(\tau)\right], \\
& \mathbb{E}\left[\Delta R_{j, u}(\tau) \Delta V_{x}(\tau) \mid \mathcal{G}(\tau)\right],
\end{aligned}
$$

for $u, x \in[D]$ and $j, z \in[r]$. Note that we do not compute second order moments taken across different time instants. As in Appendix A, we omit the conditionality on $\mathcal{G}(\tau)$ and, to compute the expectations in (68)-(70), we average over all possible positions where a degree-one check node can be removed. For instance,

$$
\begin{aligned}
& \mathbb{E}\left[\Delta R_{j, u}(\tau) \Delta R_{z, x}(\tau)\right] \\
& =\sum_{m=1}^{D} \mathbb{E}\left[\Delta R_{j, u}(\tau) \Delta R_{z, x}(\tau) \mid \operatorname{pos}(\tau)=m\right] p_{m}(\tau) .
\end{aligned}
$$

To evaluate $\mathbb{E}\left[\Delta R_{j, u}(\tau) \Delta R_{z, x}(\tau) \mid \operatorname{pos}(\tau)=m\right]$, we consider two different scenarios: $u \neq x$ and $u=x$. Namely, second order moments across different positions and within the same position. 


\section{A. Different positions. Case $u \neq x$}

First, if $u \neq x$, then it is simple to check that

$$
\mathbb{E}\left[\Delta V_{u}(\tau) \Delta V_{x}(\tau)\right]=0,
$$

since only one variable node is removed from the graph and it cannot belong to two distinct positions. Assume now that $x=u+c$, where $c$ is a strictly positive integer. If $c \geq l$, then the DD at positions $u$ and $x$ cannot be modified at the same time because check nodes at two positions further away $l-1$ positions do not share any variable node. Thus, for $c \geq l$

$$
\begin{aligned}
\mathbb{E}\left[\Delta R_{j, u}(\tau) \Delta R_{z, x}(\tau) \mid \operatorname{pos}(\tau)\right. & =m]=0, \\
\mathbb{E}\left[\Delta R_{j, u}(\tau) \Delta V_{x}(\tau) \mid \operatorname{pos}(\tau)\right. & =m]=0
\end{aligned}
$$

for any $m \in[D]$. For $c<l$, we consider the following two sub cases:

1) $\operatorname{pos}(\tau)=m, u \neq m, x \neq m$ : The probability that the DD at positions $u$ and $x$ is simultaneously modified when we remove a degree-one check node from $m$ is

$$
\xi_{m, u, x}(\tau)=\sum_{i=x-(l-1)}^{u} \lambda_{m, i}(\tau) .
$$

and, therefore,

$$
\begin{aligned}
& \mathbb{E}\left[\Delta R_{j, u}(\tau) \Delta R_{z, x}(\tau) \mid \operatorname{pos}(\tau)=m\right]= \\
& j z \xi_{m, u, x}(\tau)\left(\frac{R_{j+1, u}(\tau)-R_{j, u}(\tau)}{\sum_{q=1}^{r} R_{q, u}(\tau)}\right) \\
& \times\left(\frac{R_{z+1, x}(\tau)-R_{z, x}(\tau)}{\sum_{q=1}^{r} R_{q, x}(\tau)}\right)
\end{aligned}
$$

for $j, z<r$. For $j=r(z=r)$, we delete from (76) the term in $j+1(z+1)$. Regarding the expected product $\Delta R_{j, u}(\tau) \Delta V_{x}(\tau)$, if $c<l$ then a variable node at position $x$ is connected to a check node at position $u$ with probability one. With probability $\lambda_{m, x}(\tau)$ the variable removed along with the degree-one check node at $m$ belongs to position $x$. Consequently,

$$
\begin{aligned}
& \mathbb{E}\left[\Delta R_{j, u}(\tau) \Delta V_{x}(\tau) \mid \operatorname{pos}(\tau)=m\right] \\
& =-j \lambda_{m, x}(\tau) \frac{R_{j+1, u}(\tau)-R_{j, u}(\tau)}{\sum_{q=1}^{r} R_{q, u}(\tau)} .
\end{aligned}
$$

2) $\operatorname{pos}(\tau)=u, u \neq x$ : Since we remove a degree-one check node from position $u$, then $\Delta R_{j, u}(\tau)=0$ for any $j>1$ :

$$
\mathbb{E}\left[\Delta R_{j, u}(\tau) \Delta R_{z, x}(\tau) \mid \operatorname{pos}(\tau)=u x\right]=0
$$

for $z \in[r]$ and $j>1$. For $j=1$ we get

$$
\begin{aligned}
& \mathbb{E}\left[\Delta R_{1, u}(\tau) \Delta R_{z, x}(\tau) \mid \operatorname{pos}(\tau)=u\right] \\
& =-z \xi_{u, x}(\tau) \frac{R_{z+1, x}(\tau)-R_{z, x}(\tau)}{\sum_{q=1}^{r} R_{q, x}(\tau)},
\end{aligned}
$$

where for $z=r$ the term $R_{z+1, x}(\tau)$ in (79) is zero. Similarly

$$
\mathbb{E}\left[\Delta R_{j, u}(\tau) \Delta V_{x}(\tau) \mid \operatorname{pos}(\tau)=u\right]=0
$$

for $j>1$ and

$$
\mathbb{E}\left[\Delta R_{1, u}(\tau) \Delta V_{x}(\tau) \mid \operatorname{pos}(\tau)=u\right]=\lambda_{u, x}(\tau) .
$$

\section{B. Same positions. Case $x=u$}

Our goal now is to compute moments of the form

$$
\mathbb{E}\left[\Delta R_{j, u}(\tau) \Delta R_{z, u}(\tau)\right]
$$

for any position $u \in[D]$ and degrees $j, z \in[r]$. Assume with no loss of generality that $z \geq j$. The procedure is similar to that followed in Appendix A First we assume that the position $m$ where we remove the degree-one check node is not position $u$, i.e., $\operatorname{pos}(\tau)=m \neq u$. After deleting the variable node, one edge is removed from a check at position $u$ with probability $\xi_{m, u}(\tau)$. Besides, with probability

$$
\frac{R_{j, u}(\tau)}{\sum_{q=1}^{r} R_{q, u}(\tau)}
$$

the check node that was connected to such edge is of degree $j$. In this case, $j$ edges of right degree $j$ are removed from the graph at position $u$ and $j-1$ edges of right degree $j-1$ are created at the same position. The rest of the DD terms are not affected. Therefore,

$$
\mathbb{E}\left[\Delta R_{j, u}(\tau) \Delta R_{z, u}(\tau) \mid \operatorname{pos}(\tau)=m\right]=0, \quad z>j+1 .
$$

If $z=j+1$ we get

$$
\begin{gathered}
\mathbb{E}\left[\Delta R_{j, u}(\tau) \Delta R_{j+1, u}(\tau) \mid \operatorname{pos}(\tau)=m\right] \\
=-j(j+1) \xi_{m, u}(\tau) \frac{R_{j+1, u}(\tau)}{\sum_{u=1}^{r} R_{q, u}(\tau)},
\end{gathered}
$$

and for $j=z$ we get

$\mathbb{E}\left[\Delta R_{j, u}(\tau)^{2} \mid \operatorname{pos}(\tau)=m\right]=j^{2} \xi_{m, u}(\tau) \frac{R_{j+1, u}(\tau)+R_{j, u}(\tau)}{\sum_{u=1}^{r} R_{q, u}(\tau)}$.

For $j=r$, 85) is also equal to zero while in 86 the term $R_{j+1, u}(\tau)$ is set to zero. Finally, we compute

$$
\begin{aligned}
& \mathbb{E}\left[\Delta^{2} V_{u}(\tau) \mid \operatorname{pos}(\tau)=m\right]=\lambda_{m, u}(\tau), \\
& \mathbb{E}\left[\Delta R_{j, u}(\tau) \Delta V_{u}(\tau) \mid \operatorname{pos}(\tau)=m\right] \\
& =-j \lambda_{m, u}(\tau) \frac{R_{j+1, u}(\tau)-R_{j, u}(\tau)}{\sum_{q=1}^{r} R_{q, u}(\tau)},
\end{aligned}
$$

where for the case $j=r$ we remove the term $R_{j+1, u}(\tau)$ from the equation above.

Now we assume $\operatorname{pos}(\tau)=u$. In this case, only one edge with right degree one is removed from position $u$. Then,

$$
\mathbb{E}\left[\Delta R_{j, u}(\tau) \Delta R_{z, u}(\tau) \mid \operatorname{pos}(\tau)=u\right]=0
$$

if $j>1$ or $z>1$. For $z=j=1$ :

$$
\mathbb{E}\left[\Delta R_{1, u}(\tau)^{2} \mid \operatorname{pos}(\tau)=u\right]=1 .
$$

\section{APPENDIX C}

\section{COVARIANCE INITIAL CONDITIONS}

In Section III-B, we computed the expected DD of the $(l, r, L)$ graph after the PD initialization step, $\mathbb{E}\left[V_{u}(0)\right]$ and $\mathbb{E}\left[R_{j, u}(0)\right]$ for $j \in[r]$ and $u \in[D]$. We are now interested in computing the initial conditions for the covariance evolution equations described in Section IV namely $\operatorname{Var}\left[R_{j, u}(0)\right]$, 
$\operatorname{CoVar}\left[R_{j, u}(0), R_{z, x}(0)\right], \operatorname{Var}\left[V_{u}(0)\right], \operatorname{CoVar}\left[V_{u}(0), V_{x}(0)\right]$ and $\operatorname{CoVar}\left[V_{u}(0), R_{j, x}(0)\right]$ for $(j, z) \in[r]^{2}$ and $(u, x) \in[D]^{2}$. For simplicity, we will consider positions $u, x$ that belong both to the range $[l, \ldots, L]$, where all check nodes in the original $(l, r, L)$ graph are of degree $r$. The rest of moments, involving positions at the boundaries, can be computing in a similar procedure.

After PD initialization, the number of check nodes of degree $1,2, \ldots, r$ at position $u \in[l, L]$ is described by a multinomial distribution with $\frac{l}{r} M$ trials and probabilities

$$
p_{j, u}=\left(\begin{array}{c}
r \\
j
\end{array}\right) \epsilon^{j}(1-\epsilon)^{r-j}
$$

for $j \in[r]$, see Section $[\mathrm{III}-\mathrm{B}$. Therefore,

$$
\begin{aligned}
\operatorname{Var}\left[R_{j, u}(0)\right] & =j^{2} \frac{l}{r} M p_{j, u}\left(1-p_{j, u}\right), \\
\operatorname{CoVar}\left[R_{j, u}(0), R_{z, u}(0)\right] & =-j z \frac{l}{r} M p_{j, u} p_{z, u} .
\end{aligned}
$$

In addition, since variable nodes are independently erased

$$
\begin{aligned}
\operatorname{Var}\left[V_{u}(0)\right] & =M \epsilon(1-\epsilon), \\
\operatorname{CoVar}\left[V_{u}(0), V_{x}(0)\right] & =0 \quad u \neq x .
\end{aligned}
$$

Now we focus on the computation of moments of the form

$$
\operatorname{CoVar}\left[R_{j, u}(0), R_{z, x}(0)\right]
$$

for $x \neq u$. First, if positions $|u-x| \geq l$ then by construction of the $(l, r, L)$ ensemble a variable node cannot be connected simultaneously to one check node at position $u$ and to one check node at position $x$. Consequently, $\operatorname{CoVar}\left[R_{j, u}(0), R_{z, x}(0)\right]=$ 0 for any pair of degrees $(j, z)$ as long as $|u-x| \geq l$. For a similar reason, if $|u-x| \geq l$ then $\operatorname{CoVar}\left[V_{u}(0), R_{j, x}(0)\right]=0$.

The main idea to evaluate (96) when $|u-x|<l$ is to compute the probability that any pair of check nodes (one at position $u$ and one at position $x$ ) selected at random share at least one variable node in the $(l, r, L)$ code graph, i.e., before PD initialization. In such a case, the corresponding degrees of both check nodes after the PD initialization are not statistically independent from each other. Consider $x=u+c$ where $c<l$. There are $(l-c)$ positions of the code, from position $x-l+1$ to position $u$, in which any variable node is connected with one edge to a check node at position $u$ and with one edge to a check node at position $x$. Let $\operatorname{check}_{u}$ and $\operatorname{check}_{x}$ be a pair of check nodes selected at random from positions $u$ and $x$ and let $a(b)$ represent the number of edges of the check check $u$ $\left(\operatorname{check}_{x}\right)$ that are connected to variable nodes at positions in the range $\{x-l+1, \ldots, u\}$.

Recall from the properties of the $(l, r, L)$ ensemble described in Section II that, if we picked at random one edge connected to a check node placed at position $u$, the position of the variable node connected to such edge is a uniform random variable in the set $\{u-(l-1), \ldots, u\}$. Hence, among the $r$ edges connected to $\operatorname{check}_{u}$, the number $a$ of edges that are connected to variables in the range of positions $\{x-l+1, \ldots, u\}$ is a random variable distributed according to a Binomial distribution of $r$ trials and success probability $(l-c) / l$. The same holds for $b$. Also, $a$ and $b$ are independent random variables.
For any given pair $(a, b)$, the probability that $\operatorname{check}_{u}$ and check $_{x}$ are connected to the same variable node, placed at a position in the range $\{x-l+1, \ldots, u\}$, is as follows:

$$
(l-c) \frac{a b}{M}
$$

By averaging (97) over all possible pairs $(a, b)$ we can compute the probability that $\operatorname{check}_{u}$ and $\operatorname{check}_{x}$ share one variable node:

$$
P_{S}=\frac{l-c}{M} r^{2}\left(\frac{l-c}{l}\right)^{2}
$$

In the following, we ignore the case that $\mathrm{check}_{u}$ and $\mathrm{check}_{x}$ share two variable nodes since the probability of this to happen decays by $M^{-2}$. Denote by $d_{u}$ and $d_{x}$ the degree of the check nodes check $_{u}$ and check $x$ respectively after PD initialization. The joint probability mass function of the pair $\left(d_{u}, d_{x}\right)$ can be expressed as follows:

$$
\begin{aligned}
P\left(d_{u}=j, d_{x}=z\right) & =P\left(d_{u}=j, d_{x}=z \mid \text { share }\right) P_{S} \\
& +P\left(d_{u}=j, d_{x}=z \mid \text { no share }\right)\left(1-P_{S}\right),
\end{aligned}
$$

where $P\left(d_{u}=j, d_{x}=z \mid\right.$ share $)$ represents the joint probability distribution of the degrees $d_{u}$ and $d_{x}$ when check $_{u}$ and $\operatorname{check}_{x}$ share one variable node. It is straightforward to show that

$$
\begin{aligned}
& P\left(d_{u}=j, d_{x}=z \mid \text { share }\right) \\
& =\epsilon\left[\left(\begin{array}{c}
r-1 \\
j-1
\end{array}\right) \epsilon^{j-1}(1-\epsilon)^{r-j}\left(\begin{array}{c}
r-1 \\
z-1
\end{array}\right) \epsilon^{z-1}(1-\epsilon)^{r-z}\right] \\
& +(1-\epsilon)\left[\left(\begin{array}{c}
r-1 \\
j
\end{array}\right) \epsilon^{j}(1-\epsilon)^{r-j-1}\left(\begin{array}{c}
r-1 \\
z
\end{array}\right) \epsilon^{z}(1-\epsilon)^{r-z-1}\right],
\end{aligned}
$$

and

$$
P\left(d_{u}=j, d_{x}=z \mid \text { no share }\right)=p_{j, u} p_{z, x},
$$

where $p_{j, u}$ and $p_{z, x}$ are given in 91 . Using these expressions and averaging over all possible $\left(\operatorname{check}_{u}\right.$, $\left.\operatorname{check}_{x}\right)$ pairs (there are $\left(\frac{l}{r} M\right)^{2}$ pairs in total), we obtain:

$$
\begin{aligned}
& \operatorname{CoVar}\left[R_{j, u}(0), R_{z, x=u+c}(0)\right]=j z M(l-c)^{3} \\
& \times\left(P\left(d_{u}=j, d_{x}=z \mid \text { share }\right)-P\left(d_{u}=j, d_{x}=z \mid \text { no share }\right)\right)
\end{aligned}
$$

for $u, x \in[l, \ldots, L], j, z \in[r]$ and $c<l$. By following a similar procedure, we obtain

$$
\begin{aligned}
& \operatorname{CoVar}\left[V_{u}(0), R_{j, x=u+c}(0)\right] \\
& =j M \epsilon^{j}(1-\epsilon)^{r-j}\left[\left(\begin{array}{c}
r-1 \\
j-1
\end{array}\right)-\left(\begin{array}{l}
r \\
j
\end{array}\right) \epsilon\right]
\end{aligned}
$$

for $u, x \in[l, \ldots, L]$ and $c<l$. 